\title{
Ustilago species causing leaf-stripe smut revisited
}

\author{
Julia Kruse ${ }^{1,2}$, Wolfgang Dietrich ${ }^{3}$, Horst Zimmermann ${ }^{4}$, Friedemann Klenke ${ }^{5}$, Udo Richter ${ }^{6}$, Heidrun Richter $^{6}$, and Marco Thines ${ }^{1,2,4}$
}

${ }^{1}$ Goethe University Frankfurt am Main, Faculty of Biosciences, Institute of Ecology, Evolution and Diversity, Max-von-Laue-Str. 9, D-60438 Frankfurt am Main, Germany; corresponding authors e-mail: thines@smut-fungi.net, julia.kruse@senckenberg.de

${ }^{2}$ Biodiversity and Climate Research Centre (BiK-F), Senckenberg Gesellschaft für Naturforschung, Senckenberganlage 25, D-60325 Frankfurt am Main, Germany

${ }^{3}$ Barbara-Uthmann-Ring 68, 09456 Annaberg-Buchholz, Germany

${ }^{4}$ Cluster for Integrative Fungal Research (IPF), Georg-Voigt-Str. 14-16, D-60325 Frankfurt am Main, Germany

${ }^{5}$ Grillenburger Str. 8 c, 09627 Naundorf, Germany

${ }^{6}$ Traubenweg 8, 06632 Freyburg / Unstrut, Germany

\begin{abstract}
Leaf-stripe smuts on grasses are a highly polyphyletic group within Ustilaginomycotina, occurring in three genera, Tilletia, Urocystis, and Ustilago. Currently more than 12 Ustilago species inciting stripe smuts are recognised. The majority belong to the Ustilago striiformis-complex, with about 30 different taxa described from 165 different plant species. This study aims to assess whether host distinct-lineages can be observed amongst the Ustilago leaf-stripe smuts using nine different loci on a representative set. Phylogenetic reconstructions supported the monophyly of the Ustilago striiformiscomplex that causes leaf-stripe and the polyphyly of other leaf-stripe smuts within Ustilago. Furthermore, smut specimens from the same host genus generally clustered together in well-supported clades that often had available species names for these lineages. In addition to already-named lineages, three new lineages were observed, and described as new species on the basis of host specificity and molecular differences: namely Ustilago jagei sp. nov. on Agrostis stolonifera, U. kummeri sp. nov. on Bromus inermis, and U. neocopinata sp. nov. on Dactylis glomerata.
\end{abstract}

Key words:

DNA-based taxonomy

host specificity

molecular species discrimination multigene phylogeny

new taxa

species complex

Ustilaginaceae

Article info: Submitted: 13 January 2018; Accepted: 12 March 2018; Published: 20 March 2018.

\section{INTRODUCTION}

The term "stripe smut" is commonly used to refer to Ustilaginomycotina species that cause dark brown to black linear sori of varying length in the leaves of grasses (Poaceae). Black spore masses are released after the spores have matured beneath the epidermis in the mesophyll of the host leaves (Fischer 1953, Vánky 2012). The spore release process of sori is unknown, but may be facilitated either by the withering of dead epidermal cells or by enzymatic action, as in the white blister rusts (Heller \& 2009). Of the smut genera that infect grasses, Ustilago is the most prevalent (Stoll et al. 2003, 2005, Vánky 2012).

The term stripe smut does not reflect phylogenetic relatedness, as at least two other genera, Tilletia and Urocystis, contain species that manifest similar symptoms. The vast majority of leaf-stripe smuts belong to Ustilago, including $U$. agropyri, U. bahuichivoensis, U. bethelii, U. calamagrostidis, $U$. calcarea, U. davisii, U. deyeuxiicola, U. echinata, U. filiformis, U. phlei, U. scrobiculata, U. serpens s. lat., U. sporoboli-indici, $U$. striiformis s. lat., U. trebouxii, U. trichoneurana, and U. ulei (Vánky 2012). Of these species, U. striiformis s. lat., with the type species described on Holcus lanatus, is a complex occurring on 164 species of Poaceae representing 44 different genera
(Achnatherum, Agropyron, Agrostis, Alopecurus, Ammophila, Anthoxanthum, Arctagrostis, Arrhenatherum, Avena, Beckmannia, Brachypodium, Briza, Bromus, Calamagrostis, Cleistogenes, Cynosurus, Dactylis, Danthonia, Deschampsia, Deyeuxia, Elymus, Festuca, Helictotrichon, Hierochloë, Holcus, Hordeum, Hystrix, Koeleria, Leymus, Lolium, Melica, Milium, Pennisetum, Phalaris, Phleum, Piptatherum, Poa, Polypogon, Puccinellia, Sesleria, Setaria, Sitanion, Trisetaria, and Trisetum). Based on host specificity and minor differences in spore size and surface ornamentation, approximately 30 different taxa have been described in the $U$. striiformis species complex on various host plants (Vánky 2012, Savchenko et al. 2014a). Ustilago serpens probably represents an overlooked species complex, occuring on five host genera: Agropyron, Brachypodium, Bromus, Elymus, and Leymus. Whether other species with large warts on their spores also belong to this complex, such as $U$. echinata and $U$. scrobiculata, is currently unclear.

Ustilago striiformis s. lat. on Alopecurus pratensis has often been the sole representative of this group in phylogenetic analyses (Stoll et al. 2005, Begerow et al. 2006, McTaggart et al. 2012a). Stoll et al. (2005) supported the recognition of $U$. calamagrostidis, a parasite of several species of Calamagrostis, as separate from $U$. striiformis.

Cㅇ 2018 International Mycological Association

You are free to share - to copy, distribute and transmit the work, under the following conditions:

Attribution: $\quad$ You must attribute the work in the manner specified by the author or licensor (but not in any way that suggests that they endorse you or your use of the work).

Non-commercial: $\quad$ You may not use this work for commercial purposes.

No derivative works: You may not alter, transform, or build upon this work.

For any reuse or distribution, you must make clear to others the license terms of this work, which can be found at http://creativecommons.org/licenses/by-nc-nd/3.0/legalcode. Any of the above conditions can be waived if you get permission from the copyright holder. Nothing in this license impairs or restricts the author's moral rights. 
The morphological difference was mainly in spore size and ornamentation. Savchenko et al. (2014a) provided a more detailed analysis of the $U$. striifomis species complex using several host-fungus combinations and phylogenetic reconstructions based on the nrITS and partial LSU regions. However, while two additional species were proposed as distinct in the $U$. striiformis-complex, the phylogenetic resolution was too low to draw further conclusions regarding host specificity and potential species boundaries. To resolve undescribed lineages within this species complex, Savchenko et al. (2014a) suggested that several additional gene loci and host-fungus combinations should be included. However, in line with Vánky (2012), Savchenko et al. (2014a) suggested that it would be difficult to distinguish between these lineages based on morphological characters. DNAbased characteristics, such as diagnostic SNPs, along with host specificity might be a solution towards characterizing and describing previously-named and new species (Denchev et al. 2009, Piątek et al. 2013). The aim of this study was to use a multigene phylogeny to infer the phylogenetic differentiation in the leaf stripe smuts in the genus Ustilago, particularly those in the $U$. striiformis species complex.

\section{MATERIAL AND METHODS}

\section{Plant and fungal material}

Specimens used in the study are listed in Table 1. The names of the hosts and fungi was derived from the latest version of The International Plant Names Index (www.ipni.org), Index Fungorum (www.indexfungorum.org/) and Vánky (2012), and partly following a broad generic concept for Ustilago (Thines 2016). A majority of the samples were collected in Germany (about 76) and most collections were not older than 20 years. Samples are deposited in Herbarium Senckenbergianum Görlitz (GLM). All host identifications were confirmed by ITS sequences.

\section{DNA extraction and PCR}

About 2-20 mg of infected plant tissue was taken from fungarium samples, placed in $2 \mathrm{~mL}$ plastic reaction tubes and homogenized in a mixer mill (MM2, Retsch) using a combination of three to five $1 \mathrm{~mm}$ and two $3 \mathrm{~mm}$ metal beads at $25 \mathrm{~Hz}$ for 5-10 min. Genomic DNA was extracted using the BioSprint 96 DNA Plant Kit (Qiagen, Hilden) loaded to a KingFisher Flex robot (Thermo Scientific, Dreieich).

The complete nrITS of all DNA extracts were amplified using PCR following the procedure of White et al. (1990). The primer pairs M-ITS1 (Stoll et al. 2003) / ITS4 (White et al. 1990) or M-ITS1 / smITS-R1 (Kruse et al. 2017a) were used as the reverse and forward primers, respectively. For DNA samples from historic specimens, including type specimens, the Ustilaginaceae-optimised reverse primer ITS-US3R (5'TATCAAAACCCGGCAGGGAAG3'), located at the ITS2 region, was used.

The NL1 and NL4 primer pair (O'Donnell 1993) were used to amplify the Large Subunit (LSU) of the nrDNA with an annealing temperature of $53{ }^{\circ} \mathrm{C}$. For other loci, the following regions were amplified with their respective primer pairs and annealing temperatures in brackets: myosin R0.5/F3 $\left(55^{\circ} \mathrm{C}\right)$, map R6/F2 $\left(56{ }^{\circ} \mathrm{C}\right)$, rp/3 R1/F1 $\left(53^{\circ} \mathrm{C}\right)$, tif2 R3/F3 $\left(53^{\circ} \mathrm{C}\right)$, $s s c 1 \mathrm{R} 1 / \mathrm{F} 2\left(53^{\circ} \mathrm{C}\right)$, sdh1 R3/F2 $\left(53^{\circ} \mathrm{C}\right), r p / 4 A \mathrm{R} 1 / \mathrm{F} 4\left(53^{\circ} \mathrm{C}\right)$, and atp2 R4/F6 $\left(53^{\circ} \mathrm{C}\right)$ (Kruse et al. 2017b).

The plant ITS was amplified using the primer pair ITS1P and ITS4 (Ridgway et al. 2003) at $53{ }^{\circ} \mathrm{C}$ annealing temperature. The cycling reaction was performed in a thermocycler (Eppendorf Mastercycler 96 vapo protect; Eppendorf, Hamburg) with an initial denaturation at $95{ }^{\circ} \mathrm{C}$ for $4 \mathrm{~min}, 36 \mathrm{PCR}$ cycles of denaturation at $95{ }^{\circ} \mathrm{C}$ for 40 $\mathrm{s}$, annealing between $53-56{ }^{\circ} \mathrm{C}$ (depending on the specific primer pair) for $40 \mathrm{~s}$ and elongation at $72{ }^{\circ} \mathrm{C}$ for $60 \mathrm{~s}$, followed by a final elongation at $72{ }^{\circ} \mathrm{C}$ for $4 \mathrm{~min}$. For DNA samples older than 50 years, PCR cycles were increased to 46 cycles and a larger amount of DNA $(1.5 \mu \mathrm{L}$ of extracted DNA in a reaction volume of $11 \mu \mathrm{L}$ ) was used. The resulting amplicons were sequenced at the Biodiversity and Climate Research Centre (BiK-F) laboratory using the abovementioned PCR primers. However, amplicons from M-ITS1/smITS-R1 were sequenced using the ITS4 reverse primer. The resulting sequences were deposited in GenBank (https://www.ncbi. nlm.nih.gov/genbank/, Table 1).

\section{Alignments and phylogenetic tree reconstruction}

We used 93 samples (including 62 of the Ustilago striiformis -complex) for the phylogenetic analysis; 93 had sequences from nrlTS, atp2 (ATP synthase subunit 2) and ssc1 (member of the heat shock proteins), and 70 had additional sequences from myosin (myosin group I), map (methionine aminopeptidase), rp/3 (ribosomal protein L3), tif2 (initial translation factor of elF4A), $s d h 1$ (succinate dehydrogenase ubiquinone flavoprotein), and rp/4A (ribosomal protein L4-A) (Table 1). About two thirds of the samples (62) belonged to the $U$. striiformis species complex. Sporisorium inopinatum (syn. Langdonia inopinata) was chosen as outgroup, according to the findings of McTaggart et al. (2012a).

Alignments were made on individual loci using mafft v. 7 (Katoh \& Standley 2013) using the G-INS-i algorithm. Both leading and trailing gaps of the alignments were removed manually. Two different sets of concatenated alignments for the phylogenetic constructions were generated. The first multigene-alignment includes three loci (ITS, atp2, and ssc1) from 93 smut samples. The resulting total alignment was 1502 bp (ITS: 643 bp, atp2: 595 bp, ssc1: 264 bp). The second multigene-alignment included nine genes with a final alignment of 3156 bp (ITS: 643 bp, atp2: 595 bp, ssc1 264 bp, map: 251 bp, myosin: 257 bp, rp/4A: 415 bp, rp/3: 218 bp, sdh1: 269 bp, tif2: 244 bp).

The diagnostic bases for the $U$. striiformis species complex for all gene markers were determined using the above mentioned alignments. One further ITS alignment was created (443 bp), with the sequence of the type specimen of $U$. bromina (Table 1 ), the $U$. bromina sequences from GenBank (KF381006-8) and sequences from the same hostfungus-combination from this study, to check if all specimens were sequence-identical with the type collection of $U$. bromina on Bromus inermis (data not shown).

For phylogenetic tree constructions, Minimum Evolution (ME) analysis was done using Mega 6.06 (Tamura et al. 2013) with the Tamura-Nei substitution model and assuming 


\begin{tabular}{|c|c|c|c|c|c|c|c|c|c|c|c|c|c|c|c|}
\hline \multirow[b]{2}{*}{ DNA-no. } & \multirow[b]{2}{*}{ Species } & \multirow[b]{2}{*}{ Host } & \multicolumn{4}{|c|}{ Collection details } & \multicolumn{9}{|c|}{ gene loci } \\
\hline & & & Location & Date & Collector & $\begin{array}{l}\text { Fungarium } \\
\text { no. }\end{array}$ & ITS & atp2 & ssc1 & map & myosin & rpl4A & rpl3 & sdh1 & tif2 \\
\hline 2354 & $\begin{array}{l}\text { Sporisorium } \\
\text { aff. inopiatum } \\
\text { (Langdonia) }\end{array}$ & $\begin{array}{l}\text { Aristida } \\
\text { adscensionis }\end{array}$ & Zambia & 12 Apr. 2001 & $\begin{array}{l}\text { C., T. \& K. } \\
\text { Vánky }\end{array}$ & M-0215944 & $\begin{array}{l}\text { Kruse et } \\
\text { al. } 2017 \mathrm{~b}\end{array}$ & $\begin{array}{l}\text { Kruse et al. } \\
2017 \mathrm{~b}\end{array}$ & $\begin{array}{l}\text { Kruse et } \\
\text { al. 2017b }\end{array}$ & $\begin{array}{l}\text { Kruse et } \\
\text { al. 2017b }\end{array}$ & $\begin{array}{l}\text { Kruse et al. } \\
2017 \mathrm{~b}\end{array}$ & $\begin{array}{l}\text { Kruse et } \\
\text { al. 2017b }\end{array}$ & KY929824 & KY929964 & KY930127 \\
\hline 474 & $\begin{array}{l}\text { Ustilago } \\
\text { agrostidis- } \\
\text { palustris }\end{array}$ & $\begin{array}{l}\text { Agrostis cf. } \\
\text { gigantea }\end{array}$ & $\begin{array}{l}\text { Germany, } \\
\text { Bavaria }\end{array}$ & 22 Jun. 2012 & J. Kruse & GLM-F105832 & KY929551 & KY930157 & KY929994 & KY929709 & KY929639 & KY929849 & KY929779 & KY929919 & KY930082 \\
\hline 1374 & & $\begin{array}{l}\text { Agrostis } \\
\text { gigantea }\end{array}$ & Switzerland & 9 Jul. 2004 & V. Kummer & GLM-F107425 & KY929582 & KY930188 & KY930025 & KY929729 & KY929659 & KY929869 & KY929799 & KY929939 & KY930102 \\
\hline 2395 & & Agrostis sp. & $\begin{array}{l}\text { Germany, } \\
\text { Lower Saxony }\end{array}$ & 12 Jul. 2014 & $\begin{array}{l}\text { J. Kruse \& H. } \\
\text { Jage }\end{array}$ & GLM-F107439 & KY929596 & KY930202 & KY930039 & KY929739 & KY929669 & KY929879 & KY929809 & KY929949 & KY930112 \\
\hline 2287 & $\begin{array}{l}\text { Ustilago airae- } \\
\text { caespitosae }\end{array}$ & $\begin{array}{l}\text { Deschampsia } \\
\text { caespitosa }\end{array}$ & Polen & 13 Jul. 1994 & H. Scholz & В 700014901 & KY929526 & KY930132 & KY929969 & KY929688 & KY929618 & KY929828 & KY929758 & KY929898 & KY930061 \\
\hline 2401 & & $\begin{array}{l}\text { Deschampsia } \\
\text { caespitosa }\end{array}$ & $\begin{array}{l}\text { Austria, Upper } \\
\text { Austria }\end{array}$ & 15 Aug. 2014 & J. Kruse & GLM-F107444 & KY929601 & KY930207 & KY930044 & KY929744 & KY929674 & KY929884 & KY929814 & KY929954 & KY930117 \\
\hline 2402 & & $\begin{array}{l}\text { Deschampsia } \\
\text { caespitosa }\end{array}$ & $\begin{array}{l}\text { Austria, Upper } \\
\text { Austria }\end{array}$ & 15 Aug. 2014 & J. Kruse & GLM-F107445 & KY929602 & KY930208 & KY930045 & KY929745 & KY929675 & KY929885 & KY929815 & KY929955 & KY930118 \\
\hline 477 & $\begin{array}{l}\text { Ustilago } \\
\text { alopecurivora }\end{array}$ & $\begin{array}{l}\text { Alopecurus } \\
\text { pratensis }\end{array}$ & $\begin{array}{l}\text { Germany, } \\
\text { Hesse }\end{array}$ & 22 May 2010 & J. Kruse & GLM-F105834 & KY929553 & KY930159 & KY929996 & KY929711 & KY929641 & KY929851 & KY929781 & KY929921 & KY930084 \\
\hline 1376 & & $\begin{array}{l}\text { Alopecurus } \\
\text { pratensis }\end{array}$ & $\begin{array}{l}\text { Germany, } \\
\text { Saxony-Anhalt }\end{array}$ & 20 May 2013 & H. Jage & GLM-F107426 & KY929583 & KY930189 & KY930026 & - & - & - & - & - & - \\
\hline 1822 & $\begin{array}{l}\text { Ustilago aff. } \\
\text { andropogonis } \\
\text { (Sporisorium) }\end{array}$ & $\begin{array}{l}\text { Bothriochloa } \\
\text { ischaemum }\end{array}$ & $\begin{array}{l}\text { Germany, } \\
\text { Saxony-Anhalt }\end{array}$ & 25 Jul. 2004 & $\begin{array}{l}\text { H. Jage \& H. } \\
\text { John }\end{array}$ & GLM-F062665 & $\begin{array}{l}\text { Kruse et } \\
\text { al. 2017b }\end{array}$ & $\begin{array}{l}\text { Kruse et al. } \\
2017 \mathrm{~b}\end{array}$ & $\begin{array}{l}\text { Kruse } e t \\
\text { al. } 2017 \mathrm{~b}\end{array}$ & $\begin{array}{l}\text { Kruse et } \\
\text { al. } 2017 \mathrm{~b}\end{array}$ & $\begin{array}{l}\text { Kruse et al. } \\
2017 \mathrm{~b}\end{array}$ & $\begin{array}{l}\text { Kruse et } \\
\text { al. 2017b }\end{array}$ & KY929764 & KY929904 & KY930067 \\
\hline 432 & $\begin{array}{l}\text { Ustilago } \\
\text { perennans }\end{array}$ & $\begin{array}{l}\text { Arrhenatherum } \\
\text { elatius }\end{array}$ & $\begin{array}{l}\text { Germany, } \\
\text { Schleswig- } \\
\text { Holstein }\end{array}$ & 21 Jun. 2007 & J. Kruse & GLM-F105817 & KY929536 & KY930142 & KY929979 & KY929697 & KY929627 & KY929837 & KY929767 & KY929907 & KY930070 \\
\hline 2398 & Ustilago brizae & Briza media & Austria, Tirol & 21 Jul. 2014 & J. Kruse & GLM-F107442 & $\begin{array}{l}\text { Kruse et } \\
\text { al. 2017b }\end{array}$ & $\begin{array}{l}\text { Kruse et al. } \\
2017 \mathrm{~b}\end{array}$ & $\begin{array}{l}\text { Kruse et } \\
\text { al. 2017b }\end{array}$ & $\begin{array}{l}\text { Kruse et } \\
\text { al. 2017b }\end{array}$ & $\begin{array}{l}\text { Kruse et al. } \\
2017 \mathrm{~b}\end{array}$ & $\begin{array}{l}\text { Kruse et } \\
\text { al. 2017b }\end{array}$ & KY929812 & KY929952 & KY930115 \\
\hline 2399 & & Briza media & $\begin{array}{l}\text { Germany, } \\
\text { Bavaria }\end{array}$ & 19 Jul. 2014 & J. Kruse & GLM-F107443 & KY929600 & KY930206 & KY930043 & KY929743 & KY929673 & KY929883 & KY929813 & KY929953 & KY930116 \\
\hline 498 & $\begin{array}{l}\text { Ustilago } \\
\text { bromina }\end{array}$ & Bromus inermis & $\begin{array}{l}\text { Germany, } \\
\text { Saxony-Anhalt }\end{array}$ & 04 Jun. 2011 & J. Kruse & GLM-F105843 & $\begin{array}{l}\text { Kruse } e t \\
\text { al. 2017b }\end{array}$ & $\begin{array}{l}\text { Kruse et al. } \\
2017 \mathrm{~b}\end{array}$ & $\begin{array}{l}\text { Kruse et } \\
\text { al. 2017b }\end{array}$ & $\begin{array}{l}\text { Kruse et } \\
\text { al. } 2017 \mathrm{~b}\end{array}$ & $\begin{array}{l}\text { Kruse et al. } \\
2017 \mathrm{~b}\end{array}$ & $\begin{array}{l}\text { Kruse et } \\
\text { al. 2017b }\end{array}$ & KY929785 & KY929925 & KY930088 \\
\hline 500 & & Bromus inermis & $\begin{array}{l}\text { Germany, } \\
\text { Thuringia }\end{array}$ & 15 Jun. 2013 & J. Kruse & GLM-F105844 & KY929563 & KY930169 & KY930006 & KY929716 & KY929646 & KY929856 & KY929786 & KY929926 & KY930089 \\
\hline 1180 & & Bromus inermis & $\begin{array}{l}\text { Germany, } \\
\text { Berlin }\end{array}$ & May 1983 & H. Scholz & $\begin{array}{l}\text { HUV No } 498 \\
\text { (TUB) }\end{array}$ & KY929613 & KY930219 & KY930056 & - & - & - & - & - & - \\
\hline 2070 & & $\begin{array}{l}\text { Bromus } \\
\text { inermis }\end{array}$ & $\begin{array}{l}\text { Germany, } \\
\text { Berlin }\end{array}$ & Aug. 1892 & P. Sydow & В 700014775 & KY929525 & - & - & - & - & - & - & - & - \\
\hline 2275 & & Bromus inermis & $\begin{array}{l}\text { Germany, } \\
\text { Brandenburg }\end{array}$ & 17 Jul. 2005 & H. \& I. Scholz & В 700014755 & KY929524 & KY930131 & KY929968 & - & - & - & - & - & - \\
\hline
\end{tabular}


ARTICLE

Table 1. (Continued)

\begin{tabular}{|c|c|c|c|c|c|c|c|c|c|c|c|c|c|c|c|}
\hline \multirow[b]{2}{*}{ DNA-no. } & \multirow[b]{2}{*}{ Species } & \multirow[b]{2}{*}{ Host } & \multicolumn{4}{|c|}{ Collection details } & \multicolumn{9}{|c|}{ gene loci } \\
\hline & & & Location & Date & Collector & $\begin{array}{l}\text { Fungarium } \\
\text { no. }\end{array}$ & ITS & atp2 & ssc1 & map & myosin & rpl4A & rpl3 & sdh1 & tif2 \\
\hline 2276 & & Bromus inermis & $\begin{array}{l}\text { Germany, } \\
\text { Thuringia }\end{array}$ & 10 Sep. 1999 & I. Scholz & В 700021843 & KY929527 & KY930133 & KY929970 & - & - & - & - & - & - \\
\hline 1591 & $\begin{array}{l}\text { Ustilago aff. } \\
\text { bromivora }\end{array}$ & Bromus rigidus & Greece & 23 Apr. 2013 & C. \& F. Klenke & GLM-F107429 & KY929586 & KY930192 & KY930029 & KY929731 & KY929661 & KY929871 & KY929801 & KY929941 & KY930104 \\
\hline 3370 & & Bromus sterilis & $\begin{array}{l}\text { Spain, } \\
\text { Andalusia }\end{array}$ & 2 May 2015 & J. Kruse & GLM-F107449 & $\begin{array}{l}\text { Kruse et } \\
\text { al. } 2017 \mathrm{~b}\end{array}$ & $\begin{array}{l}\text { Kruse et al. } \\
2017 \mathrm{~b}\end{array}$ & $\begin{array}{l}\text { Kruse et } \\
\text { al. 2017b }\end{array}$ & $\begin{array}{l}\text { Kruse et } \\
\text { al. 2017b }\end{array}$ & $\begin{array}{l}\text { Kruse et al. } \\
2017 \mathrm{~b}\end{array}$ & $\begin{array}{l}\text { Kruse et } \\
\text { al. 2017b }\end{array}$ & KY929818 & KY929958 & KY930121 \\
\hline 442 & $\begin{array}{l}\text { Ustilago } \\
\text { calamagrostidis }\end{array}$ & $\begin{array}{l}\text { Calamagrostis } \\
\text { epigejos }\end{array}$ & $\begin{array}{l}\text { Germany, } \\
\text { Lower Saxony }\end{array}$ & 03 Aug. 2011 & J. Kruse & GLM-F105818 & KY929537 & KY930143 & KY929980 & - & - & - & - & - & - \\
\hline 445 & & $\begin{array}{l}\text { Calamagrostis } \\
\text { epigejos }\end{array}$ & $\begin{array}{l}\text { Germany, } \\
\text { Baden- } \\
\text { Württemberg }\end{array}$ & 20 Jul. 2013 & J. Kruse & GLM-F105819 & $\begin{array}{l}\text { Kruse et } \\
\text { al. 2017b }\end{array}$ & $\begin{array}{l}\text { Kruse et al. } \\
2017 \mathrm{~b}\end{array}$ & $\begin{array}{l}\text { Kruse et } \\
\text { al. 2017b }\end{array}$ & $\begin{array}{l}\text { Kruse et } \\
\text { al. 2017b }\end{array}$ & $\begin{array}{l}\text { Kruse et al. } \\
2017 \mathrm{~b}\end{array}$ & $\begin{array}{l}\text { Kruse et } \\
\text { al. 2017b }\end{array}$ & KY929768 & KY929908 & KY930071 \\
\hline 1383 & & $\begin{array}{l}\text { Calamagrostis } \\
\text { epigejos }\end{array}$ & $\begin{array}{l}\text { Germany, } \\
\text { Saxony-Anhalt }\end{array}$ & 29 Jun. 2013 & $\begin{array}{l}\text { H. } \\
\text { Zimmermann, } \\
\text { U. Richter }\end{array}$ & GLM-F107427 & KY929584 & KY930190 & KY930027 & KY929730 & KY929660 & KY929870 & KY929800 & KY929940 & KY930103 \\
\hline 1912 & & $\begin{array}{l}\text { Calamagrostis } \\
\text { epigejos }\end{array}$ & $\begin{array}{l}\text { Germany, } \\
\text { Saxony-Anhalt }\end{array}$ & 09 Aug. 1996 & H. Jage & GLM-F048100 & KY929530 & KY930136 & KY929973 & KY929691 & KY929621 & KY929831 & KY929761 & KY929901 & KY930064 \\
\hline 1182 & $\begin{array}{l}\text { Ustilago } \\
\text { corcontica }\end{array}$ & $\begin{array}{l}\text { Calamagrostis } \\
\text { villosa }\end{array}$ & $\begin{array}{l}\text { Germany, } \\
\text { Saxony }\end{array}$ & 22 Aug. 1987 & W. Dietrich & $\begin{array}{l}\text { HUV No } 794 \\
\text { (TUB) }\end{array}$ & KY929615 & KY930221 & KY930058 & - & - & - & - & - & - \\
\hline 1611 & & $\begin{array}{l}\text { Calamagrostis } \\
\text { villosa }\end{array}$ & $\begin{array}{l}\text { Germany, } \\
\text { Saxony-Anhalt }\end{array}$ & 26 Jul. 2003 & H. \& U. Richter & GLM-F107434 & KY929591 & KY930197 & KY930034 & - & - & - & - & - & - \\
\hline 1825 & $\begin{array}{l}\text { Ustilago cruenta } \\
\text { (Sporisorium) }\end{array}$ & $\begin{array}{l}\text { Sorghum } \\
\text { bicolor }\end{array}$ & Greece & 11 May 2006 & H-W, Otto & GLM-F078871 & $\begin{array}{l}\text { Kruse et } \\
\text { al. 2017b }\end{array}$ & $\begin{array}{l}\text { Kruse et al. } \\
2017 \mathrm{~b}\end{array}$ & $\begin{array}{l}\text { Kruse et } \\
\text { al. 2017b }\end{array}$ & $\begin{array}{l}\text { Kruse et } \\
\text { al. 2017b }\end{array}$ & $\begin{array}{l}\text { Kruse et al. } \\
2017 \mathrm{~b}\end{array}$ & $\begin{array}{l}\text { Kruse et } \\
\text { al. 2017b }\end{array}$ & KY929766 & KY929906 & KY930069 \\
\hline 3375 & $\begin{array}{l}\text { Ustilago } \\
\text { cynodontis }\end{array}$ & $\begin{array}{l}\text { Cynodon } \\
\text { dactylon }\end{array}$ & $\begin{array}{l}\text { Spain, } \\
\text { Andalusia }\end{array}$ & 3 May 2015 & J. Kruse & GLM-F107450 & KY929607 & KY930213 & KY930050 & KY929749 & KY929679 & KY929889 & KY929819 & KY929959 & KY930122 \\
\hline 3376 & & $\begin{array}{l}\text { Cynodon } \\
\text { dactylon }\end{array}$ & $\begin{array}{l}\text { Spain, } \\
\text { Andalusia }\end{array}$ & 3 May 2015 & J. Kruse & GLM-F107451 & KY929608 & KY930214 & KY930051 & KY929750 & KY929680 & KY929890 & KY929820 & KY929960 & KY930123 \\
\hline 1596 & $\begin{array}{l}\text { Ustilago aff. } \\
\text { dactyloctaenii } \\
\text { (Sporisorium) }\end{array}$ & $\begin{array}{l}\text { Dactyloctenium } \\
\text { australe }\end{array}$ & South-Africa & 22 Feb. 2000 & V. Kummer & GLM-F107430 & KY929587 & KY930193 & KY930030 & KY929732 & KY929662 & KY929872 & KY929802 & KY929942 & KY930105 \\
\hline 478 & $\begin{array}{l}\text { Ustilago } \\
\text { denotarisii }\end{array}$ & $\begin{array}{l}\text { Arrhenatherum } \\
\text { elatius }\end{array}$ & $\begin{array}{l}\text { Germany, } \\
\text { Schleswig- } \\
\text { Holstein }\end{array}$ & 13 May 2007 & J. Kruse & GLM-F105835 & KY929554 & KY930160 & KY929997 & - & - & - & - & - & - \\
\hline 481 & & $\begin{array}{l}\text { Arrhenatherum } \\
\text { elatius }\end{array}$ & $\begin{array}{l}\text { Germany, } \\
\text { Rhineland- } \\
\text { Palatinate }\end{array}$ & 23 May 2010 & J. Kruse & GLM-F105836 & KY929555 & KY930161 & KY929998 & - & - & - & - & - & - \\
\hline 483 & & $\begin{array}{l}\text { Arrhenatherum } \\
\text { elatius }\end{array}$ & $\begin{array}{l}\text { Germany, } \\
\text { Lower Saxony }\end{array}$ & 31 Jul. 2011 & J. Kruse & GLM-F105837 & KY929556 & KY930162 & KY929999 & - & - & - & - & - & - \\
\hline 486 & & $\begin{array}{l}\text { Arrhenatherum } \\
\text { elatius }\end{array}$ & $\begin{array}{l}\text { Germany, } \\
\text { Thuringia }\end{array}$ & 04 Jun. 2012 & J. Kruse & GLM-F105838 & KY929557 & KY930163 & KY930000 & - & - & - & - & - & - \\
\hline 488 & & $\begin{array}{l}\text { Arrhenatherum } \\
\text { elatius }\end{array}$ & $\begin{array}{l}\text { Germany, } \\
\text { Bavaria }\end{array}$ & 16 May 2013 & J. Kruse & GLM-F105839 & KY929558 & KY930164 & KY930001 & - & - & - & - & - & - \\
\hline
\end{tabular}




\begin{tabular}{|c|c|c|c|c|c|c|c|c|c|c|c|c|c|c|c|}
\hline \multirow[b]{2}{*}{ DNA-no. } & \multirow[b]{2}{*}{ Species } & \multirow[b]{2}{*}{ Host } & \multicolumn{4}{|c|}{ Collection details } & \multicolumn{9}{|c|}{ gene loci } \\
\hline & & & Location & Date & Collector & $\begin{array}{l}\text { Fungarium } \\
\text { no. }\end{array}$ & ITS & atp2 & ssc1 & map & myosin & rpl4A & $\mathrm{rpl3}$ & sdh1 & tif2 \\
\hline 447 & $\begin{array}{l}\text { Ustilago } \\
\text { echinata }\end{array}$ & $\begin{array}{l}\text { Phalaris } \\
\text { arundinacea }\end{array}$ & $\begin{array}{l}\text { Germany, } \\
\text { Lower Saxony }\end{array}$ & 01 Jul. 2010 & J. Kruse & GLM-F105820 & KY929539 & KY930145 & KY929982 & KY929699 & KY929629 & KY929839 & KY929769 & KY929909 & KY930072 \\
\hline 449 & & $\begin{array}{l}\text { Phalaris } \\
\text { arundinacea }\end{array}$ & $\begin{array}{l}\text { Germany, } \\
\text { Lower Saxony }\end{array}$ & 29 Aug. 2011 & J. Kruse & GLM-F105821 & KY929540 & KY930146 & KY929983 & KY929700 & KY929630 & KY929840 & KY929770 & KY929910 & KY930073 \\
\hline 1914 & & $\begin{array}{l}\text { Phalaris } \\
\text { arundinacea }\end{array}$ & $\begin{array}{l}\text { Switzerland, } \\
\text { St. Gallen }\end{array}$ & 26 Jul. 2000 & H. Jage & GLM-F048338 & KY929531 & KY930137 & KY929974 & KY929692 & KY929622 & KY929832 & KY929762 & KY929902 & KY930065 \\
\hline 451 & $\begin{array}{l}\text { Ustilago aff. } \\
\text { filiformis }\end{array}$ & $\begin{array}{l}\text { Glyceria } \\
\text { fluitans }\end{array}$ & $\begin{array}{l}\text { Germany, } \\
\text { Lower Saxony }\end{array}$ & 17 May 2007 & J. Kruse & GLM-F105822 & KY929541 & KY930147 & KY929984 & KY929701 & KY929631 & KY929841 & KY929771 & KY929911 & KY930074 \\
\hline 454 & & $\begin{array}{l}\text { Glyceria } \\
\text { fluitans }\end{array}$ & $\begin{array}{l}\text { Germany, } \\
\text { Bavaria }\end{array}$ & 24 Jun. 2012 & J. Kruse & GLM-F105823 & KY929542 & KY930148 & KY929985 & KY929702 & KY929632 & KY929842 & KY929772 & KY929912 & KY930075 \\
\hline 455 & & $\begin{array}{l}\text { Glyceria } \\
\text { fluitans }\end{array}$ & $\begin{array}{l}\text { Germany, } \\
\text { Bavaria }\end{array}$ & 10 May 2013 & J. Kruse & GLM-F105824 & $\begin{array}{l}\text { Kruse et } \\
\text { al. } 2017 \mathrm{~b}\end{array}$ & $\begin{array}{l}\text { Kruse et al. } \\
2017 \mathrm{~b}\end{array}$ & $\begin{array}{l}\text { Kruse et } \\
\text { al. } 2017 \mathrm{~b}\end{array}$ & $\begin{array}{l}\text { Kruse et } \\
\text { al. 2017b }\end{array}$ & $\begin{array}{l}\text { Kruse et al. } \\
2017 \mathrm{~b}\end{array}$ & $\begin{array}{l}\text { Kruse et } \\
\text { al. 2017b }\end{array}$ & KY929773 & KY929913 & KY930076 \\
\hline 456 & $\begin{array}{l}\text { Ustilago } \\
\text { filiformis }\end{array}$ & $\begin{array}{l}\text { Glyceria } \\
\text { maxima }\end{array}$ & $\begin{array}{l}\text { Germany, } \\
\text { Lower Saxony }\end{array}$ & 01 Jul. 2010 & J. Kruse & GLM-F105825 & KY929544 & KY930150 & KY929987 & KY929704 & KY929634 & KY929844 & KY929774 & KY929914 & KY930077 \\
\hline 472 & $\begin{array}{l}\text { Ustilago jagei } \\
\text { sp. nov. }\end{array}$ & $\begin{array}{l}\text { Agrostis } \\
\text { rupestris }\end{array}$ & $\begin{array}{l}\text { Switzerland, } \\
\text { Grisons }\end{array}$ & 02 Aug. 2009 & J. Kruse & GLM-F105830 & KY929549 & KY930155 & KY929992 & - & - & - & - & - & - \\
\hline 473 & & $\begin{array}{l}\text { Agrostis } \\
\text { stolonifera }\end{array}$ & $\begin{array}{l}\text { Germany, } \\
\text { Bavaria }\end{array}$ & 20 May 2012 & J. Kruse & GLM-F105831 & KY929550 & KY930156 & KY929993 & - & - & - & - & - & - \\
\hline 476 & & $\begin{array}{l}\text { Agrostis } \\
\text { stolonifera }\end{array}$ & $\begin{array}{l}\text { Germany, } \\
\text { Hesse }\end{array}$ & 22 May 2010 & J. Kruse & GLM-F105833 & KY929552 & KY930158 & KY929995 & KY929710 & KY929640 & KY929850 & KY929780 & KY929920 & KY930083 \\
\hline 551 & & Agrostis sp. & $\begin{array}{l}\text { Germany, } \\
\text { Lower Saxony }\end{array}$ & 11 Jun. 2010 & J. Kruse & GLM-F107423 & KY929580 & KY930186 & KY930023 & KY929727 & KY929657 & KY929867 & KY929797 & KY929937 & KY930100 \\
\hline 2396 & & $\begin{array}{l}\text { Agrostis } \\
\text { stolonifera }\end{array}$ & $\begin{array}{l}\text { Germany, } \\
\text { Bavaria }\end{array}$ & 20 Jul. 2014 & J. Kruse & GLM-F107440 & KY929597 & KY930203 & KY930040 & KY929740 & KY929670 & KY929880 & KY929810 & KY929950 & KY930113 \\
\hline 2397 & & $\begin{array}{l}\text { Agrostis } \\
\text { stolonifera }\end{array}$ & $\begin{array}{l}\text { Germany, } \\
\text { Hesse }\end{array}$ & 27 Jun. 2014 & J. Kruse & GLM-F107441 & KY929598 & KY930204 & KY930041 & KY929741 & KY929671 & KY929881 & KY929811 & KY929951 & KY930114 \\
\hline 494 & & Agrostis sp. & $\begin{array}{l}\text { Germany, } \\
\text { Bavaria }\end{array}$ & 04 Jul. 2013 & J. Kruse & GLM-F105841 & KY929560 & KY930166 & KY930003 & KY929713 & KY929643 & KY929853 & KY929783 & KY929923 & KY930086 \\
\hline 1375 & & $\begin{array}{l}\text { Agrostis } \\
\text { stolonifera }\end{array}$ & $\begin{array}{l}\text { Germany, } \\
\text { Saxony- } \\
\text { Anhalt }\end{array}$ & 16 Sep. 2001 & H. Jage & GLM-F047379 & KY929528 & KY930134 & KY929971 & KY929689 & KY929619 & KY929829 & KY929759 & KY929899 & KY930062 \\
\hline 1612 & $\begin{array}{l}\text { Ustilago } \\
\text { kummeri sp. } \\
\text { nov. }\end{array}$ & $\begin{array}{l}\text { Bromus } \\
\text { inermis }\end{array}$ & $\begin{array}{l}\text { Germany, } \\
\text { Brandenburg }\end{array}$ & 19 Jun. 2010 & V. Kummer & GLM-F107435 & KY929592 & KY930198 & KY930035 & KY929736 & KY929666 & KY929876 & KY929806 & KY929946 & KY930109 \\
\hline 1948 & & Bromus inermis & $\begin{array}{l}\text { Germany, } \\
\text { Saxony-Anhalt }\end{array}$ & 17 Jul. 2001 & $\begin{array}{l}\text { H. Jage, W. } \\
\text { Lehman }\end{array}$ & GLM-F047380 & KY929529 & KY930135 & KY929972 & KY929690 & KY929620 & KY929830 & KY929760 & KY929900 & KY930063 \\
\hline 501 & Ustilago Ioliicola & Lolium perenne & $\begin{array}{l}\text { Germany, } \\
\text { Bavaria }\end{array}$ & 14 May 2013 & J. Kruse & GLM-F105845 & KY929564 & KY930170 & KY930007 & - & - & - & - & - & - \\
\hline 2288A & & $\begin{array}{l}\text { Festuca } \\
\text { pratensis }\end{array}$ & $\begin{array}{l}\text { Germany, } \\
\text { Hesse }\end{array}$ & 25 May 2014 & J. Kruse & GLM-F107437 & KY929594 & KY930200 & KY930037 & - & - & - & - & - & - \\
\hline
\end{tabular}


Table 1. (Continued)

\begin{tabular}{|c|c|c|c|c|c|c|c|c|c|c|c|c|c|c|c|}
\hline \multirow[b]{2}{*}{ DNA-no. } & \multirow[b]{2}{*}{ Species } & \multirow[b]{2}{*}{ Host } & \multicolumn{4}{|c|}{ Collection details } & \multicolumn{9}{|c|}{ gene loci } \\
\hline & & & Location & Date & Collector & $\begin{array}{l}\text { Fungarium } \\
\text { no. }\end{array}$ & ITS & atp2 & ssc1 & map & myosin & rpl4A & $\mathrm{rpl} 3$ & sdh1 & tif2 \\
\hline 3386 & & $\begin{array}{l}\text { Festuca } \\
\text { arundinacea }\end{array}$ & $\begin{array}{l}\text { Germany, } \\
\text { Hesse }\end{array}$ & 02 Nov. 2014 & J. Kruse & GLM-F107454 & KY929611 & KY930217 & KY930054 & KY929753 & KY929683 & KY929893 & KY929823 & KY929963 & KY930126 \\
\hline 2815A & Ustilago maydis & Zea mays & $\begin{array}{l}\text { Germany, } \\
\text { Saxony-Anhalt }\end{array}$ & 10 Jul. 2007 & H. Jage & GLM-F107446 & KY929603 & KY930209 & KY930046 & KY929746 & KY929676 & KY929886 & KY929816 & KY929956 & KY930119 \\
\hline 1404 & Ustilago milii & Milium effusum & $\begin{array}{l}\text { Germany, } \\
\text { Saxony-Anhalt }\end{array}$ & 02 Jun. 2002 & H. Jage & GLM-F107428 & KY929585 & KY930191 & KY930028 & - & - & - & - & - & - \\
\hline 2303 & & Milium effusum & $\begin{array}{l}\text { Germany, } \\
\text { Saxony }\end{array}$ & 03 Jun. 2012 & W. Dietrich & GLM-F107438 & KY929595 & KY930201 & KY930038 & KY929738 & KY929668 & KY929878 & KY929808 & KY929948 & KY930111 \\
\hline 3385 & & Milium effusum & $\begin{array}{l}\text { Germany, } \\
\text { Hesse }\end{array}$ & 11 Jun. 2015 & J. Kruse & GLM-F107453 & KY929610 & KY930216 & KY930053 & KY929752 & KY929682 & KY929892 & KY929822 & KY929962 & KY930125 \\
\hline 503 & $\begin{array}{l}\text { Ustilago } \\
\text { neocopinata sp. } \\
\text { nov. }\end{array}$ & $\begin{array}{l}\text { Dactylis } \\
\text { glomerata }\end{array}$ & $\begin{array}{l}\text { Germany, } \\
\text { Lower Saxony }\end{array}$ & 01 Jul. 2010 & J. Kruse & GLM-F105846 & KY929565 & KY930171 & KY930008 & - & - & - & - & - & - \\
\hline 505 & & $\begin{array}{l}\text { Dactylis } \\
\text { glomerata }\end{array}$ & $\begin{array}{l}\text { Germany, } \\
\text { Bavaria }\end{array}$ & 20 Jun. 2010 & J. Kruse & GLM-F105847 & KY929566 & KY930172 & KY930009 & - & - & - & - & - & - \\
\hline 506 & & $\begin{array}{l}\text { Dactylis } \\
\text { glomerata }\end{array}$ & $\begin{array}{l}\text { Germany, } \\
\text { Lower Saxony }\end{array}$ & 19 May 2011 & J. Kruse & GLM-F105848 & $\begin{array}{l}\text { Kruse et } \\
\text { al. 2017b }\end{array}$ & $\begin{array}{l}\text { Kruse et al. } \\
2017 b\end{array}$ & $\begin{array}{l}\text { Kruse et } \\
\text { al. 2017b }\end{array}$ & $\begin{array}{l}\text { Kruse et } \\
\text { al. 2017b }\end{array}$ & $\begin{array}{l}\text { Kruse et al. } \\
2017 \mathrm{~b}\end{array}$ & $\begin{array}{l}\text { Kruse et } \\
\text { al. 2017b }\end{array}$ & KY929787 & KY929927 & KY930090 \\
\hline 508 & & $\begin{array}{l}\text { Dactylis } \\
\text { glomerata }\end{array}$ & $\begin{array}{l}\text { Germany, } \\
\text { Bavaria }\end{array}$ & 19 Jul. 2011 & J. Kruse & GLM-F105849 & KY929568 & KY930174 & KY930011 & KY929718 & KY929648 & KY929858 & KY929788 & KY929928 & KY930091 \\
\hline 510 & & $\begin{array}{l}\text { Dactylis } \\
\text { glomerata }\end{array}$ & $\begin{array}{l}\text { Germany, } \\
\text { Bavaria }\end{array}$ & 24 May 2012 & J. Kruse & GLM-F105850 & KY929569 & KY930175 & KY930012 & KY929719 & KY929649 & KY929859 & KY929789 & KY929929 & KY930092 \\
\hline 512 & & $\begin{array}{l}\text { Dactylis } \\
\text { glomerata }\end{array}$ & $\begin{array}{l}\text { Germany, } \\
\text { Bavaria }\end{array}$ & 15 Jun. 2012 & J. Kruse & GLM-F107413 & KY929570 & KY930176 & KY930013 & - & - & - & - & - & - \\
\hline 521 & & $\begin{array}{l}\text { Dactylis } \\
\text { glomerata }\end{array}$ & $\begin{array}{l}\text { Germany, } \\
\text { Thuringia }\end{array}$ & 15 Jun. 2013 & J. Kruse & GLM-F107414 & KY929571 & KY930177 & KY930014 & - & - & - & - & - & - \\
\hline 463 & Ustilago nuda & $\begin{array}{l}\text { Hordeum } \\
\text { vulgare }\end{array}$ & $\begin{array}{l}\text { Germany, } \\
\text { Bavaria }\end{array}$ & 12 May 2012 & J. Kruse & GLM-F105826 & $\begin{array}{l}\text { Kruse et } \\
\text { al. 2017b }\end{array}$ & $\begin{array}{l}\text { Kruse et al. } \\
2017 \mathrm{~b}\end{array}$ & $\begin{array}{l}\text { Kruse et } \\
\text { al. 2017b }\end{array}$ & $\begin{array}{l}\text { Kruse et } \\
\text { al. 2017b }\end{array}$ & $\begin{array}{l}\text { Kruse et al. } \\
2017 b\end{array}$ & $\begin{array}{l}\text { Kruse et } \\
\text { al. 2017b }\end{array}$ & KY929775 & KY929915 & KY930078 \\
\hline 884 & $\begin{array}{l}\text { Sporisorium aff. } \\
\text { occidentale }\end{array}$ & $\begin{array}{l}\text { Andropogon } \\
\text { gerardii }\end{array}$ & USA & 30 Jul. 1989 & not known & $\begin{array}{l}\text { HUV No } 758 \\
\text { (TUB) }\end{array}$ & $\begin{array}{l}\text { Kruse et } \\
\text { al. 2017b }\end{array}$ & $\begin{array}{l}\text { Kruse et al. } \\
2017 \mathrm{~b}\end{array}$ & $\begin{array}{l}\text { Kruse et } \\
\text { al. 2017b }\end{array}$ & $\begin{array}{l}\text { Kruse et } \\
\text { al. 2017b }\end{array}$ & $\begin{array}{l}\text { Kruse et al. } \\
2017 \mathrm{~b}\end{array}$ & $\begin{array}{l}\text { Kruse et } \\
\text { al. 2017b }\end{array}$ & KY929825 & KY929965 & KY930128 \\
\hline 471 & Ustilago salweyi & Holcus mollis & $\begin{array}{l}\text { Germany, } \\
\text { Bavaria }\end{array}$ & 11 Jun. 2012 & J. Kruse & GLM-F105829 & KY929548 & KY930154 & KY929991 & KY929708 & KY929638 & KY929848 & KY929778 & KY929918 & KY930081 \\
\hline 489 & & Holcus mollis & $\begin{array}{l}\text { Germany, } \\
\text { Bavaria }\end{array}$ & 16 May 2013 & J. Kruse & GLM-F105840 & KY929559 & KY930165 & KY930002 & KY929712 & KY929642 & KY929852 & KY929782 & KY929922 & KY930085 \\
\hline 523 & & Holcus lanatus & $\begin{array}{l}\text { Germany, } \\
\text { Lower Saxony }\end{array}$ & 24 May 2009 & J. Kruse & GLM-F107415 & KY929572 & KY930178 & KY930015 & KY929720 & KY929650 & KY929860 & KY929790 & KY929930 & KY930093 \\
\hline 524 & & Holcus lanatus & $\begin{array}{l}\text { Germany, } \\
\text { Lower Saxony }\end{array}$ & 22 May 2010 & J. Kruse & GLM-F107416 & $\begin{array}{l}\text { Kruse et } \\
\text { al. 2017b }\end{array}$ & $\begin{array}{l}\text { Kruse et al. } \\
2017 \mathrm{~b}\end{array}$ & $\begin{array}{l}\text { Kruse et } \\
\text { al. 2017b }\end{array}$ & $\begin{array}{l}\text { Kruse et } \\
\text { al. 2017b }\end{array}$ & $\begin{array}{l}\text { Kruse et al. } \\
2017 \mathrm{~b}\end{array}$ & $\begin{array}{l}\text { Kruse et } \\
\text { al. 2017b }\end{array}$ & KY929791 & KY929931 & KY930094 \\
\hline 525 & & Holcus lanatus & $\begin{array}{l}\text { Germany, } \\
\text { Lower Saxony }\end{array}$ & 27 May 2010 & J. Kruse & GLM-F107417 & KY929574 & KY930180 & KY930017 & KY929722 & KY929652 & KY929862 & KY929792 & KY929932 & KY930095 \\
\hline 531 & & Holcus lanatus & $\begin{array}{l}\text { Germany, } \\
\text { Bavaria }\end{array}$ & 17 May 2012 & J. Kruse & GLM-F107418 & KY929575 & KY930181 & KY930018 & - & - & - & - & - & - \\
\hline
\end{tabular}




\begin{tabular}{|c|c|c|c|c|c|c|c|c|c|c|c|c|c|c|c|}
\hline \multirow[b]{2}{*}{ DNA-no. } & \multirow[b]{2}{*}{ Species } & \multirow[b]{2}{*}{ Host } & \multicolumn{4}{|c|}{ Collection details } & \multicolumn{9}{|c|}{ gene loci } \\
\hline & & & Location & Date & Collector & $\begin{array}{l}\text { Fungarium } \\
\text { no. }\end{array}$ & ITS & atp2 & ssc1 & map & myosin & rpl4A & rpl3 & sdh1 & tif2 \\
\hline 541 & & Holcus mollis & $\begin{array}{l}\text { Germany, } \\
\text { Saxony }\end{array}$ & 03 Jun. 2011 & J. Kruse & GLM-F107419 & KY929576 & KY930182 & KY930019 & KY929723 & KY929653 & KY929863 & KY929793 & KY929933 & KY930096 \\
\hline 543 & & Holcus mollis & $\begin{array}{l}\text { Germany, } \\
\text { Saxony-Anhalt }\end{array}$ & 05 Jun. 2011 & J. Kruse & GLM-F107420 & KY929577 & KY930183 & KY930020 & KY929724 & KY929654 & KY929864 & KY929794 & KY929934 & KY930097 \\
\hline 544 & & Holcus mollis & $\begin{array}{l}\text { Germany, } \\
\text { Saxony-Anhalt }\end{array}$ & 05 Jun. 2011 & J. Kruse & GLM-F107421 & KY929578 & KY930184 & KY930021 & KY929725 & KY929655 & KY929865 & KY929795 & KY929935 & KY930098 \\
\hline 545 & & Holcus mollis & $\begin{array}{l}\text { Germany, } \\
\text { Lower Saxony }\end{array}$ & 17 Aug. 2011 & J. Kruse & GLM-F107422 & KY929579 & KY930185 & KY930022 & KY929726 & KY929656 & KY929866 & KY929796 & KY929936 & KY930099 \\
\hline 497 & Ustilago scaura & $\begin{array}{l}\text { Helictotrichon } \\
\text { pubescens }\end{array}$ & $\begin{array}{l}\text { Germany, } \\
\text { Rhineland- } \\
\text { Palatinate }\end{array}$ & 23 May 2010 & J. Kruse & GLM-F105842 & KY929561 & KY930167 & KY930004 & KY929714 & KY929644 & KY929854 & KY929784 & KY929924 & KY930087 \\
\hline 3384 & & $\begin{array}{l}\text { Helictotrichon } \\
\text { pubescens }\end{array}$ & $\begin{array}{l}\text { Germany, } \\
\text { Hesse }\end{array}$ & 10 Jun. 2015 & J. Kruse & GLM-F107452 & KY929609 & KY930215 & KY930052 & KY929751 & KY929681 & KY929891 & KY929821 & KY929961 & KY930124 \\
\hline 1359 & $\begin{array}{l}\text { Ustilago aff. } \\
\text { schroeteriana } \\
\text { (Sporisorium) }\end{array}$ & $\begin{array}{l}\text { Paspalum } \\
\text { virgatum }\end{array}$ & Costa Rica & 15 Mar. 1991 & T. \& K. Vánky & $\begin{array}{l}\text { HUV No } 888 \\
\text { (TUB) }\end{array}$ & $\begin{array}{l}\text { Kruse et } \\
\text { al. 2017b }\end{array}$ & $\begin{array}{l}\text { Kruse et al. } \\
2017 \mathrm{~b}\end{array}$ & $\begin{array}{l}\text { Kruse et } \\
\text { al. 2017b }\end{array}$ & $\begin{array}{l}\text { Kruse et } \\
\text { al. 2017b }\end{array}$ & $\begin{array}{l}\text { Kruse et al. } \\
2017 \mathrm{~b}\end{array}$ & $\begin{array}{l}\text { Kruse et } \\
\text { al. 2017b }\end{array}$ & KY929826 & KY929966 & KY930129 \\
\hline 1608 & $\begin{array}{l}\text { Ustilago } \\
\text { scrobiculata }\end{array}$ & $\begin{array}{l}\text { Calamagrostis } \\
\text { epigejos }\end{array}$ & $\begin{array}{l}\text { Germany, } \\
\text { Brandenburg }\end{array}$ & 17 Aug. 2011 & $\begin{array}{l}\text { V. Kummer \& } \\
\text { C. Buhr }\end{array}$ & GLM-F107431 & KY929588 & KY930194 & KY930031 & KY929733 & KY929663 & KY929873 & KY929803 & KY929943 & KY930106 \\
\hline 1609 & & $\begin{array}{l}\text { Calamagrostis } \\
\text { epigejos }\end{array}$ & $\begin{array}{l}\text { Germany, } \\
\text { Thuringia }\end{array}$ & 27 May 2010 & V. Kummer & GLM-F107432 & KY929589 & KY930195 & KY930032 & KY929734 & KY929664 & KY929874 & KY929804 & KY929944 & KY930107 \\
\hline 1610 & & $\begin{array}{l}\text { Calamagrostis } \\
\text { epigejos }\end{array}$ & $\begin{array}{l}\text { Germany, } \\
\text { Brandenburg }\end{array}$ & 24 Jun. 2007 & V. Kummer & GLM-F107433 & $\begin{array}{l}\text { Kruse et } \\
\text { al. 2017b }\end{array}$ & $\begin{array}{l}\text { Kruse et al. } \\
2017 \mathrm{~b}\end{array}$ & $\begin{array}{l}\text { Kruse et } \\
\text { al. 2017b }\end{array}$ & $\begin{array}{l}\text { Kruse et } \\
\text { al. 2017b }\end{array}$ & $\begin{array}{l}\text { Kruse et al. } \\
2017 \mathrm{~b}\end{array}$ & $\begin{array}{l}\text { Kruse et } \\
\text { al. 2017b }\end{array}$ & KY929805 & KY929945 & KY930108 \\
\hline 467 & $\begin{array}{l}\text { Ustilago } \\
\text { serpens }\end{array}$ & Elymus repens & $\begin{array}{l}\text { Germany, } \\
\text { Schleswig- } \\
\text { Holstein }\end{array}$ & 31 Jul. 2012 & J. Kruse & GLM-F105827 & KY929546 & KY930152 & KY929989 & KY929706 & KY929636 & KY929846 & KY929776 & KY929916 & KY930079 \\
\hline 469 & & Elymus repens & $\begin{array}{l}\text { Germany, } \\
\text { Thuringia }\end{array}$ & 15 Jun. 2013 & J. Kruse & GLM-F105828 & KY929547 & KY930153 & KY929990 & KY929707 & KY929637 & KY929847 & KY929777 & KY929917 & KY930080 \\
\hline 3110 & & Elymus repens & $\begin{array}{l}\text { Germany, } \\
\text { Brandenburg }\end{array}$ & 29 Jun. 2014 & V. Kummer & GLM-F107447 & KY929604 & KY930210 & KY930047 & - & - & - & - & - & - \\
\hline 1305 & $\begin{array}{l}\text { Ustilago } \\
\text { aff. sorghi } \\
\text { (Sporisorium) }\end{array}$ & $\begin{array}{l}\text { Sorghum } \\
\text { plumosum }\end{array}$ & Australia & 20 Feb. 1996 & $\begin{array}{l}\text { A. A. Mitchell, } \\
\text { C. \& K. Vánky }\end{array}$ & $\begin{array}{l}\text { HUV No } 970 \\
\text { (TUB) }\end{array}$ & $\begin{array}{l}\text { Kruse et } \\
\text { al. 2017b }\end{array}$ & $\begin{array}{l}\text { Kruse et al. } \\
2017 \mathrm{~b}\end{array}$ & $\begin{array}{l}\text { Kruse et } \\
\text { al. 2017b }\end{array}$ & $\begin{array}{l}\text { Kruse et } \\
\text { al. 2017b }\end{array}$ & $\begin{array}{l}\text { Kruse et al. } \\
2017 \mathrm{~b}\end{array}$ & $\begin{array}{l}\text { Kruse et } \\
\text { al. 2017b }\end{array}$ & KY929827 & KY929967 & KY930130 \\
\hline 1951 & $\begin{array}{l}\text { Ustilago aff. } \\
\text { syntherismae }\end{array}$ & $\begin{array}{l}\text { Digitaria } \\
\text { sanguinalis }\end{array}$ & $\begin{array}{l}\text { Germany, } \\
\text { Saxony-Anhalt }\end{array}$ & 01 Oct . 2004 & H. Jage & GLM-F064759 & KY929534 & KY930140 & KY929977 & KY929695 & KY929625 & KY929835 & KY929765 & KY929905 & KY930068 \\
\hline 1617 & & $\begin{array}{l}\text { Digitaria } \\
\text { sanguinalis }\end{array}$ & $\begin{array}{l}\text { Germany, } \\
\text { Brandenburg }\end{array}$ & 11 Aug. 2001 & V. Kummer & GLM-F107436 & $\begin{array}{l}\text { Kruse et } \\
\text { al. 2017b }\end{array}$ & $\begin{array}{l}\text { Kruse et al. } \\
2017 \mathrm{~b}\end{array}$ & $\begin{array}{l}\text { Kruse et } \\
\text { al. 2017b }\end{array}$ & $\begin{array}{l}\text { Kruse et } \\
\text { al. 2017b }\end{array}$ & $\begin{array}{l}\text { Kruse et al. } \\
2017 \mathrm{~b}\end{array}$ & $\begin{array}{l}\text { Kruse et } \\
\text { al. } 2017 \mathrm{~b}\end{array}$ & KY929807 & KY929947 & KY930110 \\
\hline 553 & $\begin{array}{l}\text { Ustilago } \\
\text { trichophora }\end{array}$ & $\begin{array}{l}\text { Echinochloa } \\
\text { crus-galli }\end{array}$ & $\begin{array}{l}\text { Germany, } \\
\text { North Rhine- } \\
\text { Westphalia }\end{array}$ & 04 Oct . 2010 & J. Kruse & GLM-F107424 & $\begin{array}{l}\text { Kruse et } \\
\text { al. 2017b }\end{array}$ & $\begin{array}{l}\text { Kruse et al. } \\
2017 \mathrm{~b}\end{array}$ & $\begin{array}{l}\text { Kruse et } \\
\text { al. } 2017 \mathrm{~b}\end{array}$ & $\begin{array}{l}\text { Kruse et } \\
\text { al. 2017b }\end{array}$ & $\begin{array}{l}\text { Kruse et al. } \\
2017 \mathrm{~b}\end{array}$ & $\begin{array}{l}\text { Kruse et } \\
\text { al. 2017b }\end{array}$ & KY929798 & KY929938 & KY930101 \\
\hline
\end{tabular}


complete deletion at $80 \%$ cut-off with 1000 bootstrap replicates. All other parameters were set to default values. Maximum Likelihood (ML) analysis was done using RAxML (Stamatakis 2014) with parameters set to default values and Bayesian analysis was done using MrBayes 3.2 (Ronquist \& Huelsenbeck 2003) running five times with model 6 (GTR) using four incrementally heated chains for 10 million generations, sampling every $1000^{\text {th }}$ tree discarding the first $30 \%$ of the obtained trees, all other parameters were set to default on the TrEase webserver (http://www.thines-lab.senckenberg.de/trease).

To account for potentially deviating evolutionary properties, the analysis in ME was done also on a partitioned concatenated dataset. As no supported differences within the topology of the trees were observed in comparison with the un-partitioned dataset, the other analyses were carried out without partitioning.

\section{Morphological examination}

For light microscopy, fungarium specimens (GLM-F107417, GLM-F105836, GLM-F107435, GLM-F107413, GLM-F047379, GLM-F105827) were transferred to $60 \%$ lactic acid on a slide. Morphological examination was carried out using a Zeiss Imager M2 AX10 microscope (Carl Zeiss, Göttingen). Measurements of the spores were performed at $x 400$. The measurements are reported as maxima and minima in parentheses, and the mean plus and minus the standard deviation of a number of measurements is given in parenthesis. The means are placed in italics.

\section{RESULTS}

\section{Phylogenetic inference}

The LSU sequence data were excluded from further analysis since sequences were identical for all members of the Ustilago striformis species complex (data not shown). All other loci showed SNPs within the $U$. striiformis cluster. The diagnostic bases (SNPs) with their specific positions are given in Fig. 6 .

There were no supported conflicts in the topology of the trees of the single loci and the concatenated trees. Thus, the datasets were combined and used as concatenated for further analysis. The multigene tree based on nine different loci (Fig. 1) showed strong to maximum support for a monophyly of the $U$. striiformis species complex. If multiple specimens from one host species were included, these grouped together with strong to maximum support, except for the clades corresponding to $U$. scaura s. lat. (ME 64, ML 63, BA 0.99), U. brizae (ME 63, ML 68, BA 0.99), and $U$. agrostidis-palustris (ME 71, ML 68, BA 0.99), which received weak to strong support (Fig. 1).

A phylogenetic reconstruction (Fig. 2) with an additional 21 specimens but based on only half of the characters per specimen (ITS, atp2, and ssc1) revealed the same groups as the double-sized alignment, but expectedly with weaker statistical support. For example, the three weak to strongly supported lineages shown in Fig. 1 still grouped together, but with no or weak support (U. brizae - ME 64, ML -, BA 0.79; U. scaura s. lat. - ME -, ML -, BA 0.79; U. agrostidispalustris - no support), highlighting the importance of gene selection.

In the phylogenetic reconstruction based on fewer genes (Fig. 2) additional specimens were included, which further supported the high degree of genetic differentiation in conjunction with the host species infected. Specimens from Festuca and Lolium grouped together with strong support, while the monophyly of the clade containing samples from Alopecurus species was unsupported to weakly supported (ME 64, ML -, BA -). Two monophyletic groups were absent from the tree with more loci (Fig. 1): one on Calamagrostis villosa and another on Arrhenatherum elatius. Both of these groups were highly supported (Calamagrostis: ME 76, ML 94, BA 0.99; Arrhenatherum: ME 92, ML 99, BA 1) in the tree based on fewer loci (Fig. 2).

In both phylogenetic trees (Figs 1-2), U. cynodontis was inferred as the sister species to the whole $U$. striiformis species complex. To illustrate the relationships within this species complex further, two additional phylogenetic 


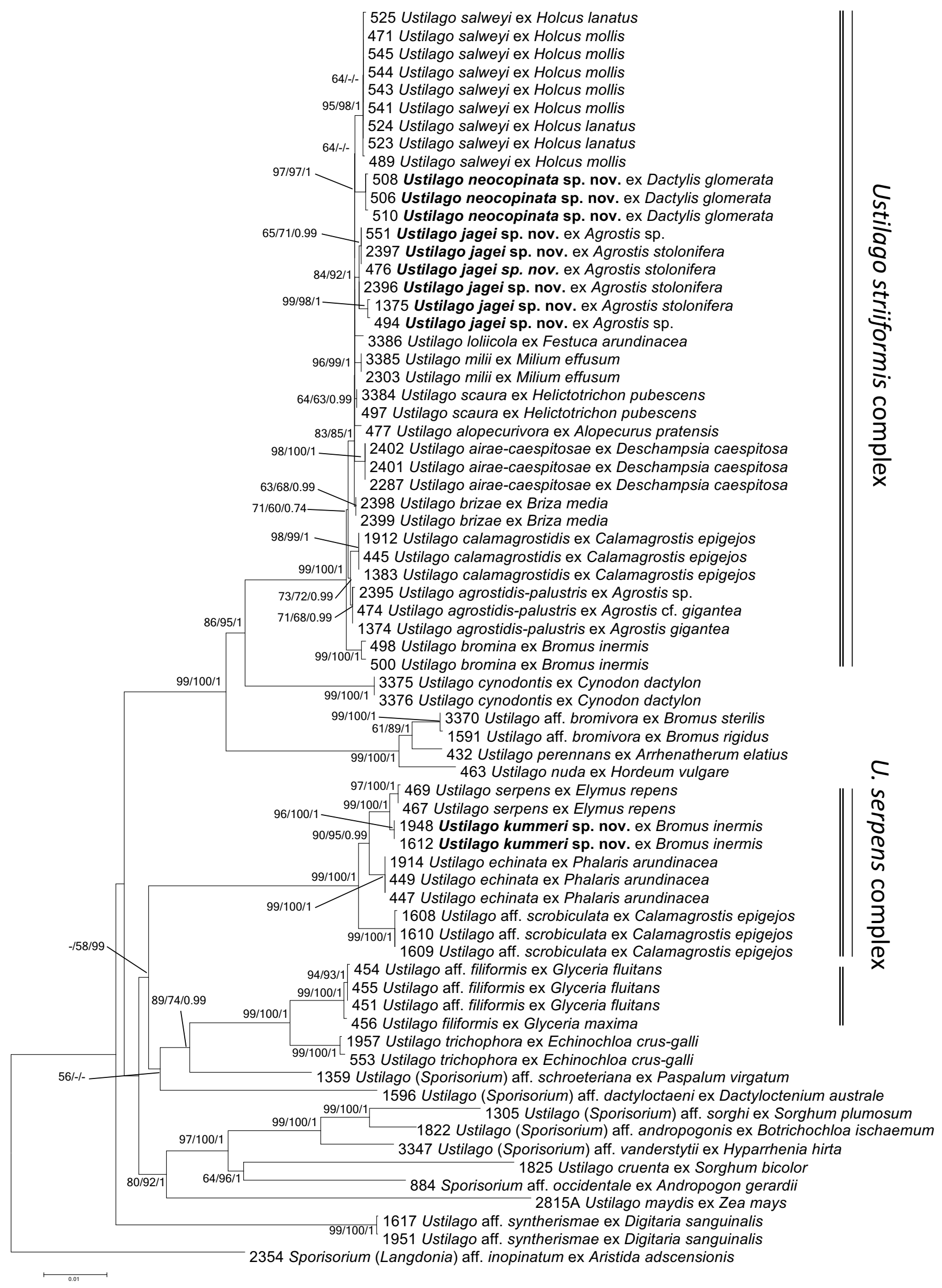

Fig. 1. Phylogenetic tree based on Minimum Evolution analysis of nine loci (ITS, myosin, map, rp/3, tif2, ssc1, sdh1, rp/4A, atp2). Numbers on branches denote support in Minimum Evolution, Maximum Likelihood and Bayesian Analyses, in the respective order. Values below $55 \%$ are denoted by ' - '. The bar indicates the number of substitutions per site. 
92/93/1 442 Ustilago calamagrostidis ex Calamagrostis epigejos 445 Ustilago calamagrostidis ex Calamagrostis epigejos 1312 Ustilago calamagrostidis ex Calamagrostis epigejos 1182 Ustilago corcontica ex Calamagrostis villosa

76/94/0.99 1611 Ustilago corcontica ex Calamagrostis villosa 474 Ustilago agrostidis-palustris ex Agrostis cf. gigantea

-I-/0.79 1374 Ustilago agrostidis-palustris ex Agrostis gigantea 2395 Ustilago agrostidis-palustris ex Agrostis sp.

473 Ustilago jagei sp. nov. ex Agrostis stolonifera

2396 Ustilago jagei sp. nov. ex Agrostis stolonifera

$-162 / 0.98-2397$ Ustilago jagei sp. nov. ex Agrostis stolonifera 472 Ustilago jagei sp. nov. ex Agrostis rupestris

476 Ustilago jagei sp. nov. ex Agrostis stolonifera

551 Ustilago jagei sp. nov. ex Agrostis sp.

1375 Ustilago jagei sp. nov. ex Agrostis stolonifera

67/75/0.99 494 Ustilago jagei sp. nov. ex Agrostis sp.

3386 Ustilago Ioliicola ex Festuca arundinacea 3386 Ustilago loliicola ex Festuca arundin

98/100/1 7501 Ustilago loliicola ex Lolium perenne

92/99/1 $\quad \begin{aligned} & \text { 2288a Ustilago loliicola ex Festuca pratensis } \\ & 478 \text { Ustilago denotarisii ex Arrhenatherum elatius } \\ & 481 \text { Ustilago denotarisii ex Arrhenatherum elatius }\end{aligned}$

483 Ustilago denotarisii ex Arrhenatherum elatius

488 Ustilago denotarisii ex Arrhenatherum elatius

486 Ustilago denotarisii ex Arrhenatherum elatius

2303 Ustilago milii ex Milium effusum

3385 Ustilago milii ex Milium effusum

$92 / 98 / 1$
1404 Ustilago milii ex Milium effusum
3384 Ustilago scaura ex Helictotrichon pubescens

497 Ustilago scaura ex Helictotrichon pubescens

525 Ustilago salweyi ex Holcus lanatus

531 Ustilago salweyi ex Holcus lanatus

541 Ustilago salweyi ex Holcus mollis

543 Ustilago salweyi ex Holcus mollis

87/85/1 - 544 Ustilago salweyi ex Holcus mollis

545 Ustilago salweyi ex Holcus mollis

523 Ustilago salweyi ex Holcus lanatus

524 Ustilago salweyi ex Holcus lanatus

489 Ustilago salweyi ex Holcus mollis

489 Ustilago salweyi ex Holcus mollis

471 ustilago salweyi ex Holcus mollis

\begin{tabular}{r|r}
2399 Ustilago brizae ex Briza media \\
1180 Ustilago bromina ex Bromus inermis \\
2275 Ustilago bromina ex Bromus inermis
\end{tabular}

2275 Ustilago bromina ex Bromus inermis

2276 Ustilago bromina ex Bromus inermis

99/99/1 498 Ustilago bromina ex Bromus inermis

521 Ustilago neocopinata sp. nov. ex Dactylis glomerata

503 Ustilago neocopinata sp. nov. ex Dactylis glomerata

87/95/1 - $\quad-505$ Ustilago neocopinata sp. nov. ex Dactylis glomerata

100/90/1 506 Ustilago neocopinata sp. nov. ex Dactylis glomerata

508 Ustilago neocopinata sp. nov. ex Dactylis glomerata

510 Ustilago neocopinata sp. nov. ex Dactylis glomerata

512 Ustilago neocopinata sp. nov. ex Dactylis glomerata

87/99/1__ 2401 Ustilago airae-caepitosae ex Deschampsia caespitosa

2402 Ustilago airae-caepitosae ex Deschampsia caespitosa

2287 Ustilago airae-caepitosae ex Deschampsia caespitosa

64/-I/-I 1376 Ustilago alopecurivora ex Alopecurus pratensis

477 Ustilago alopecurivora ex Alopecurus pratensis

\begin{tabular}{l|l}
3375 Ustilago cynodontis ex Cynodon dactylon \\
3376 Ustilago cynodontis ex Cynodon dactylon
\end{tabular}

100/100/1 1591 Ustilago aff. bromivora ex Bromus rigidus

3370 Ustilago aff. bromivora ex Bromus sterilis 432 Ustilago perennans ex Arrhenatherum elatius 95/92/1 463 Ustilago nuda ex Hordeum vulgare

-/59/0.97 88/96/0.99_ 75/71/7 3110 Ustilago serpens ex Elymus repens

85/99/1 99/98/1 469 Ustilago serpens ex Elymus repens

82/75/0.88 1612 Ustilago kummeri sp. nov. ex Bromus inermis 1948 Ustilago kummeri sp. nov. ex Bromus inermis

100/100/1 447 Ustilago echinata ex Phalaris arundinacea

97/100/1 1914 Ustilago echinata ex Phalaris arundinacea

1609 Ustilago aff. scrobiculata ex Calamagrostis epigejos

100/100/1 1610 Ustilago aff. scrobiculata ex Calamagrostis epigejos 1608 Ustilago aff. scrobiculata ex Calamagrostis epigejos

456 Ustilago filiformis ex Glyceria maxima

100/100/1 - 454 Ustilago aff. filiformis ex Glyceria fluitans

454 Ustilago aff. filiformis ex Glyceria fluitans

451 Ustilago aff. filiformis ex Glyceria fluitans

-553 Ustilago trichophora ex Echinochloa crus-galli

1957 Ustilago trichophora ex Echinochloa crus-galli

1359 Ustilago aff. schroeteriana (Sporisorium) ex Paspalum virgatum

1596 Ustilago aff. dactyloctaeni (Sporisorium) ex Dactyloctenium australe

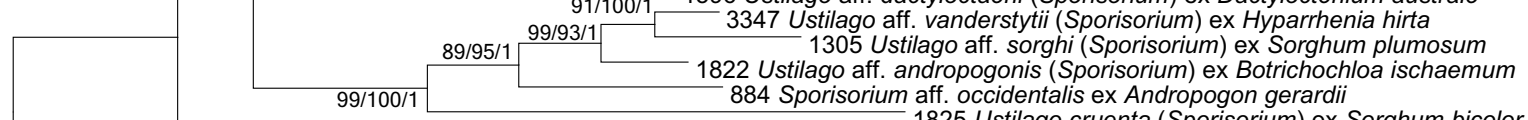

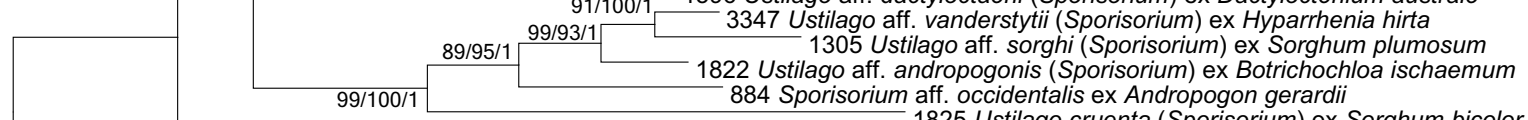
884 Sporisorium aff. occidentalis ex Andropogon gerardit

17 Ustilago aff. syntherismae ex Digitaria Sorghum bicolor

$100 / 100 / 11617$ Ustilago aff. syntherismae ex Digitaria sanguinalis 2354 Sporisorium aff. inopinatum (Langdonia) ex Aristida adscensionis

0.01

Fig. 2. Phylogenetic tree based on Minimum Evolution analysis of three loci (ITS, ssc1, atp2). Numbers on branches denote support in Minimum Evolution, Maximum Likelihood and Bayesian Analyses, in the respective order. Values below 55 \% are denoted by '-'.. The bar indicates the number of substitutions per site. 


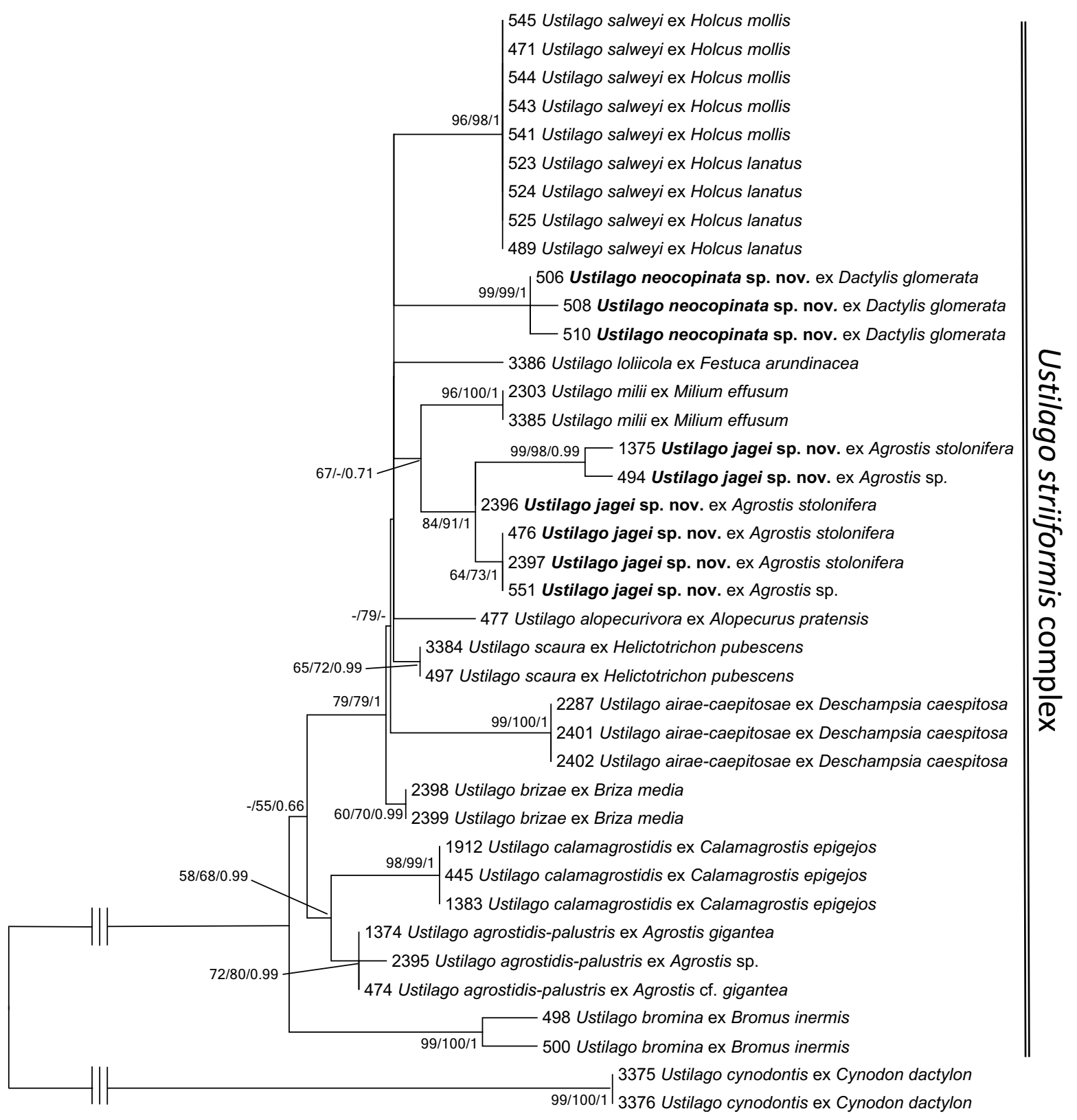

0.005

Fig. 3. Phylogenetic tree based on Minimum Evolution analysis of nine loci (ITS, myosin, map, rp/3, tif2, ssc1, sdh1, rp/4A, atp2) detailed showing the Ustilago striiformis-complex with the outgroup U. cynodontis. Numbers on branches denote support in Minimum Evolution, Maximum Likelihood and Bayesian Analyses, in the respective order. Values below $55 \%$ are denoted by ' - '. The bar indicates the number of substitutions per site.

trees with a reduced sampling and $U$. cynodontis as outgroup are shown in Figs 3 (9 loci) and 4 (3 loci). The support values and the topology were comparable to the phylogenetic reconstructions in Figs $1-2$. In both phylogenetic trees, $U$. serpens on Elymus repens and on Bromus inermis grouped together with high to maximum support. This group clustered with two further lineages with larger echinulate spores compared to the $U$. striiformis species complex, which is considered a synapomorphy of this lineage.

The resolution on the backbone was rather low, as highlighted also by the ambiguous placement of $U$. maydis, which was resolved as a sister group to the pathogens on the majority of panicoid hosts in the tree based on 9 loci (Fig. 1) with moderate to maximum support, while being inferred as a sister to the clade containing the $U$. species complex as well as the $U$. nuda species group with lacking to maximum support in the tree based on three loci (Fig. 2).

\section{Morphology}

The degree of overlap in morphological characteristics was too high in both species complexes to provide easily accessible characteristics for species delimitation (Fig. 5). The individual measurements are included in the species descriptions below and summarized in Table 3. 


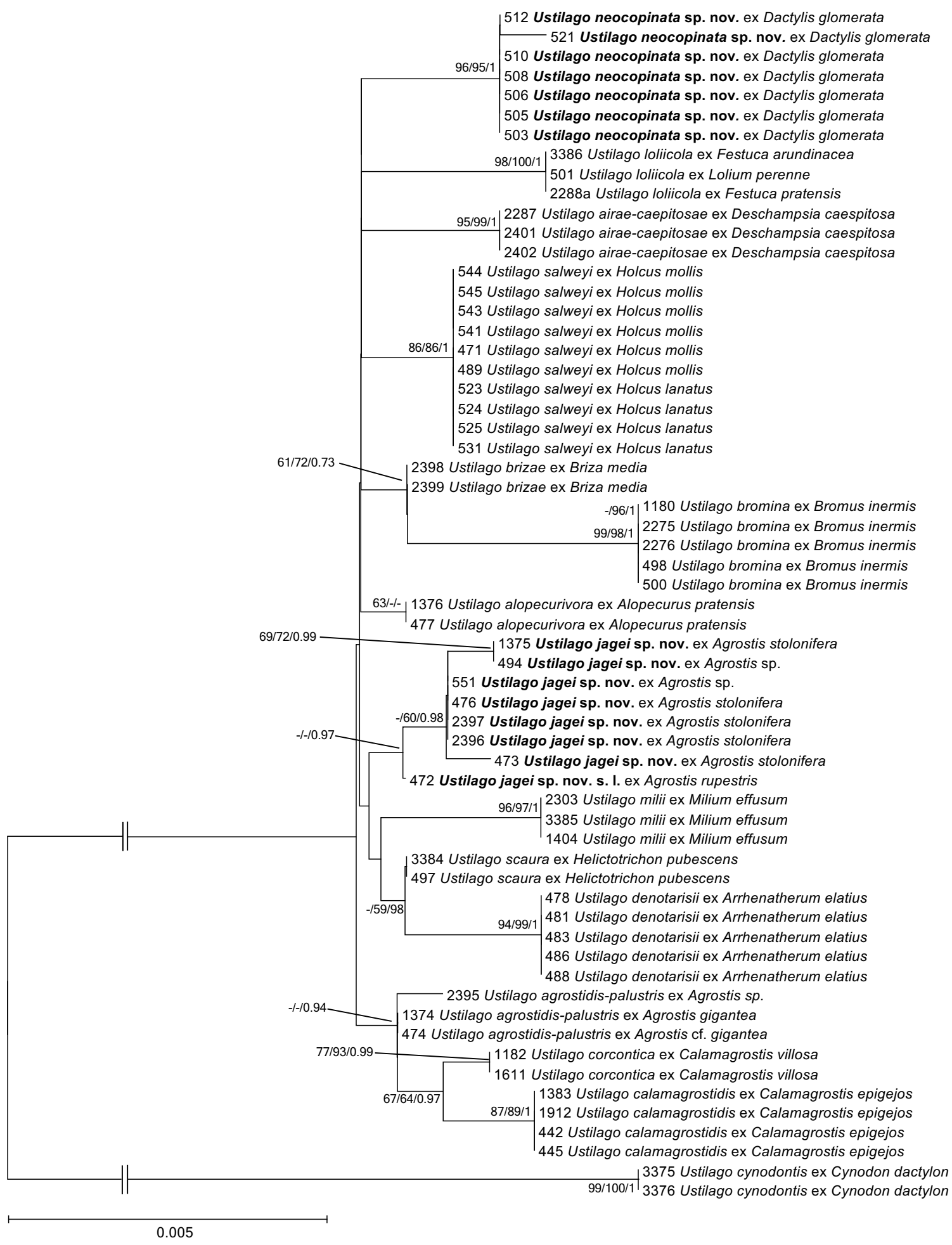

Fig. 4. Phylogenetic tree based on Minimum Evolution analysis of three loci (ITS, ssc1, atp2) detailed showing the Ustilago striiformis-complex with the outgroup U. cynodontis. Numbers on branches denote support in Minimum Evolution, Maximum Likelihood and Bayesian Analyses, in the respective order. Values below $55 \%$ are denoted by '- ${ }^{\prime}$. The bar indicates the number of substitutions per site.

\section{TAXONOMY}

Based on our phylogenetic analyses, the following nomenclature and taxonomic changes are proposed for leaf stripe smuts caused by species of Ustilago. The positions given for the diagnostic bases refer to specific positions in the alignments as highlighted in the alignment consensus sequences in Fig. 4. Only selected synonyms are given here. For a complete synonymy reference should be made to Vánky (2012) and references therein.

Ustilago agrostidis-palustris W. H. Davis ex Ciferri, Ann. Mycol. 29: 54 (1931).

Type: USA: Wisconsin: Madison, on cultivated 'redtop' (i.e. Agrostis "palustris Huds.", now Agrostis gigantea), 
8 July 1921, W. H. \& J. J. Davis (BPI 166994 - lectotype designated here, MBT 380628).

Confirmed host: Agrostis gigantea.

\section{Confirmed distribution: Germany and USA.}

Notes: Ustilago agrostidis-palustris can be distinguished from other leaf stripe smuts of the $U$. striiformis species complex based on its host specific occurrence on Agrostis gigantea s. lat. Furthermore, it differs in one diagnostic base from all other species of the $U$. striiformis-complex included in this study - in the $s d h 1$ gene there is a $\mathrm{C}$ instead of a $\mathrm{T}$ at position 138 (Table 2, Fig. 6).

Ustilago airae-caespitosae (Lindr.) Liro, Ann. Acad. Sci. Fenn., ser. A 17 (1): 71 (1924).

Basionym: Tilletia airae-caespitosae Lindr., Acta Soc. Fauna Flora Fenn. 26:15 (1904).

Type: Finland: Nyland: Helsingfors, Hagasund, on Aira caespitosa (i.e. Deschampsia caespitosa), 10 Aug. 1902, J. I. Lindroth [Vestergren, Micr. Rar. Sel. no. 806; Sydow, Ustil. no. 316] (M-0236198 - lectotype designated here, MBT 380628; from one of the several duplicate collections treated as "lectotype" by Lindeberg, Symb. Bot. Upsal. 16 (2): 135, 1959).

Confirmed host: Deschampsia caespitosa.

Confirmed distribution: Austria and Finland.

Notes: Within the Ustilago striiformis species complex, $U$. airae-caespitosae can be distinguished from other species based on the host-specific occurrence on Deschampsia caespitosa. Furthermore, it differs in six diagnostic bases from all other species within the $U$. striiformis species complex included in this study - in the atp2 gene it has an A instead of a $G$ at position 22 and 94 , in the map gene there is a $T$ instead of a $\mathrm{C}$ at position 227, in the myosin gene there is an A instead of a $G$ at position 133, in the rp/3 gene a $T$ instead of $a \mathrm{C}$ at position 199, and an A instead of a $\mathrm{G}$ at position 576 in the ITS region (Table 2, Fig. 6).

Ustilago alopecurivora (Ule) Liro, Ann. Acad. Sci. fenn., ser. A 17 (1): 72 (1924).

Basionym: Tilletia alopecurivora Ule, Hedwigia 25: 113 (1886).

Synonyms: Uredo longissima var. megalospora Riess, in Rabenhorst, Herb. Viv. Myc. no. 1897 (1854).

Ustilago megalospora (Riess) Cif., Nuovo Giorn. Bot. Ital. 40: 261 (1933).

Type: Germany: Bavaria: Coburg, Hofgarten, on Alopecurus pratensis, June 1879, E. Ule (B - holotype lost); Berlin: Charlottenburg-Nord, Kolonie Königsdamm, slope of ditch, 9 Aug. 1988, H. Scholz (B 700014985 - neotype designated here, MBT 380629).

Confirmed host: Alopecurus pratensis.

\section{Confirmed distribution: Germany.}

Notes: Within the $U$. striiformis species complex, $U$. alopecurivora can be distinguished from other species based on the host-specific occurrence on Alopecurus pratensis. Furthermore, $U$. alopecurivora differs in three diagnostic bases from all other species within the $U$. striiformis species complex included in this study - in the atp2 gene, there is an A instead of a $G$ at position 358 , in the map gene there is a $G$ instead of a T at position 192, and in the myosin gene there is a $\mathrm{T}$ instead of a C at position 83 (Table 2, Fig. 6).

Ustilago brizae (Ule) Liro, Ann. Acad. Sci. Fenn., Ser. A 17 (1): 74 (1924).

Basionym: Tilletia brizae Ule, Verh. Bot. Ver. Prov. Brandenb. 25: 214 (1884).

Type: Germany: Bavaria: Coburg, Rögener Berg, on Briza media, July 1879, E. Ule [Rabenhorst, Fungi Eur. no. 3604] (M-0147750 - lectotype designated here, MBT 380630; from one of the several duplicate collections treated as "lectotype" by Lindeberg, Symb. Bot. Upsal. 16(2): 135, 1959).

Confirmed hosts: Briza media.

Confirmed distribution: Austria and Germany.

Notes: Within the $U$. striiformis species complex, U. brizae can be distinguished from other species based on the hostspecific occurrence on Briza media. Furthermore, $U$. brizae differs in one diagnostic base from all other species within the $U$. striiformis species complex included in this study, except $U$. bromina on Bromus inermis, in having a $\mathrm{C}$ instead of a $\mathrm{T}$ at position 621 in the ITS region, and differs from $U$. bromina by having an $A$ instead of a $G$ at position 223 in the ITS region (Table 2, Fig. 6).

Ustilago corcontica (Bubák) Liro, Ann. Acad. Sci. Fenn., Ser. A 17 (1): 383 (1924).

Basionym: Tilletia corcontica Bubák, Houby Ceské, Hemibasidii 2: 47 (1912).

Type: Czech Republic: on the crest of Riesengebirge Mts, on Calamagrostis halleriana (i.e. C. villosa), 20 July 1872, J. Gerhardt (BPI 172761 - lectotype designated here, MBT 380631; one of the "isolectotypes" of Lindeberg, Symb. Bot. Upsal. 16(2): 114, 1959).

Confirmed host: Calamagrostis villosa.

Confirmed distribution: Czech Republic and Germany.

Notes: Within the U. striiformis species complex, U. corcontica can be distinguished from other species based on the hostspecific occurrence on Calamagrostis villosa. Furthermore, $U$. corcontica differs in one diagnostic bases from all other species within the $U$. striiformis species complex included in this study - in the atp2 gene there is an $T$ instead of a $C$ at position 535 (Table 2, Fig. 6). 

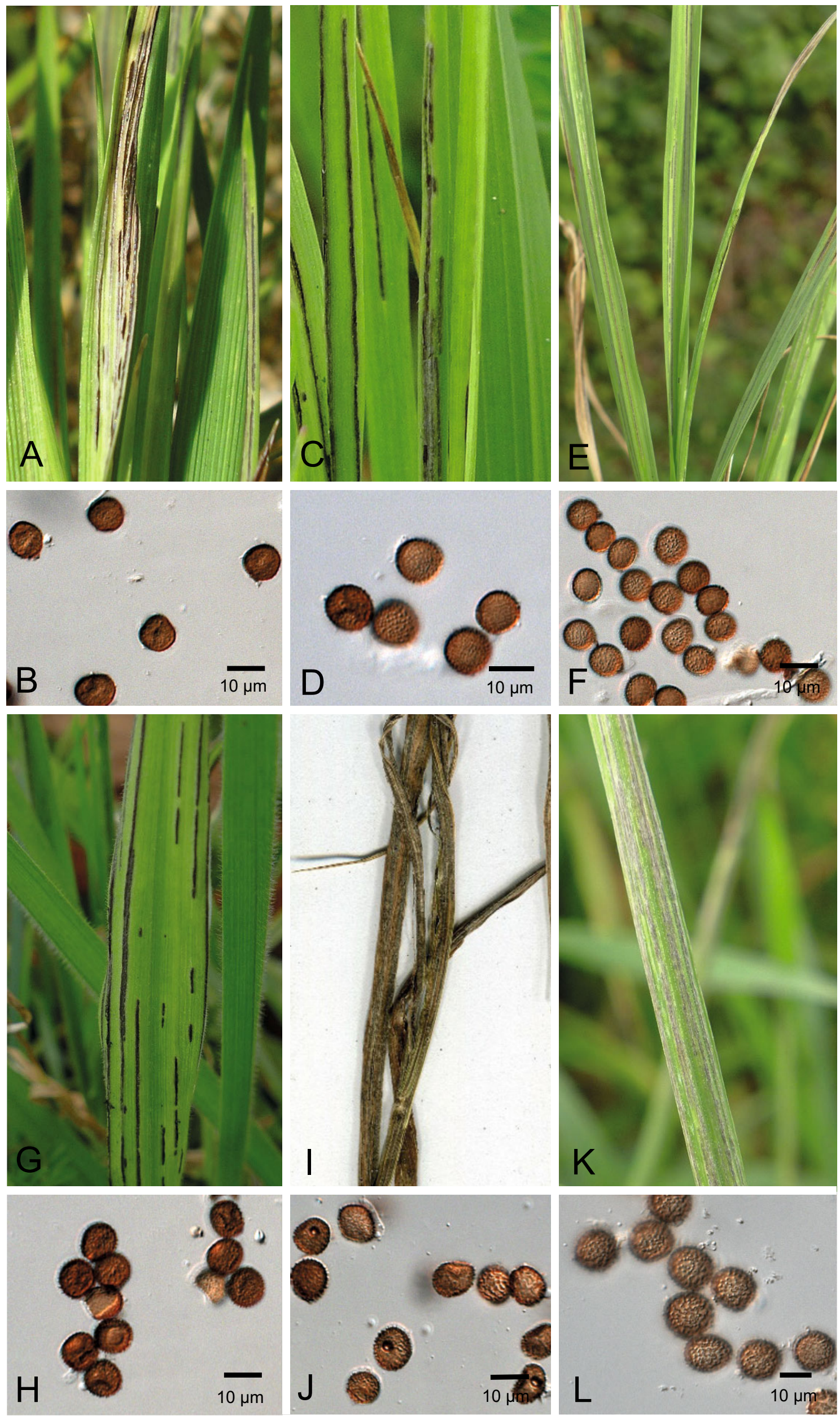
Ustilago denotarisii A. A. Fischer v. Waldheim, Aperçu Syst. Ustil.: 22 (1877); as "de Notarisii".

Type: Italy: on Arrhenatherum spp. (not located but could also not be confirmed as lost; a neotype may need to be designated for this species in the future).

Confirmed hosts: Arrhenatherum species.

Confirmed distribution: Germany and Italy.

Notes: Spores globose to ovoid, standard range (9.0-)10.5(av. 11.2)-12.0 (-13.5) × (8.0-) 9.0-(av. 9.7)-10.5(-12.0) $\mu \mathrm{m}$, length/breadth ratio of 1.10- (av. 1.20) -1.38, olive-brown, and finely echinulate. Within the $U$. striiformis species complex, $U$. denotarisii can be distinguished from other species based on the host-specific occurrence on Arrhenatherum species. Furthermore, $U$. denotarisii differs in two diagnostic bases from all other species within the $U$. striiformis species complex included in this study - in the atp2 gene there is an A instead of a $G$ at position 346 , and in the gene ssc1 there is an $\mathrm{A}$ instead of a $\mathrm{C}$ at position 182 (Table 2, Fig. 6).

Ustilago echinata J. Schröt., Abh. Schles. Ges. Vaterl. Kult., Abth. Naturwiss.: 48: 4 (1870 ["1869"].

Type: Poland: Silesia: 'Schwarzwasserbruch', near Legnica, on Phalaris arundinacea, June 1869, W. G. Schneider [Rabenhorst, Fungi Eur. no. 1497] (FR - lectotype designated here, MBT 380632; one of the several duplicate collections previously treated as "lectotype" in Rabenhorst, Fungi Eur. No. 1497).

Reported hosts: Glyceria grandis, Phalaris arundinacea, and Scolochloa festucacea.

Confirmed host: Phalaris arundinacea.

Known distribution: Asia, North America, and Europe.

Notes: This species shares one sequence motif (AACCCAAC) at positions 20-27 in the ITS region with other coarsely ornamented stripe smuts ( $U$. serpens clade in Fig. 1), and many SNPs which distinguish $U$. echinata from species of the $U$. strifformis-complex. Within the $U$. serpens-complex, $U$. echinata can be distinguished from other species based on its host-specific occurrence on Phalaris arundinacea (type host). Whether the other hosts of a similar ecotype are infected by the same species could not be clarified in the current study, but the high degree of host specificity observed in Ustilago renders it possible that specimens from other host genera will have to be described as new species. Furthermore, U. echinata differs in eight diagnostic bases from all other species within the $U$. serpens species complex included in this study - in the atp2 gene there is a G instead of an A at position 85 , in the map gene there is an $A$ instead of a $G$ at position 208, in the myosin gene there is a $\mathrm{C}$ instead of an $\mathrm{A}$ at position 141 and a $\mathrm{T}$ instead of a $C$ at position 156, in the $r p / 3$ gene there is a $T$ instead of a $C$ at position 91 and an $A$ instead of a $G$ at position 146, in the sdh 1 gene there is an $A$ instead of $a \mathrm{G}$ and at positions 58 and 256 , and in the ITS locus there is a $C$ instead of an $A$ at position 19 , a C instead of a $T$ at position 38, an A instead of a gap at position 186 and 596 and a $G$ instead of an A at positions 188 and 604 (Tab. 2, Fig. 6).

Due to the generally narrow host specificity of smut fungi, it is conceivable that $U$. echinata will be revealed to be a species group.

\section{Ustilago jagei J. Kruse \& Thines, sp. nov. MycoBank MB819627 \\ (Fig. 5A-B)}

Etymology: Named after mycologist Horst Jage from Kemberg (Germany), who has made significant contributions to the knowledge of phytopathogenic fungi and has enabled wellsampled phylogenetic investigations in various plant pathogens by his outstanding collections.

Diagnosis: Within the U. striformis species complex, U. jagei can be distinguished from other species based on its hostspecific occurrence on Agrostis stolonifera s. lat. Furthermore, $U$. jagei differs in two diagnostic bases from all other species within the $U$. striiformis species complex included in this study - in the atp2 gene there is an A instead of a $G$ at position 466 and in the gene $r p / 3$ there is an $A$ instead of a $G$ at position 92 (Table 2, Fig. 6).

Type: Germany: Saxony-Anhalt: Dessau, Kühnauer Sea, southern shore east-southeast of Großkuhnau, wayside, on Agrostis stolonifera, 16 Sept. 2001, H. Jage (GLM-F047379 - holotype).

Description: Sori as long narrow streaks parallel to vascular bundles, mostly in the leaves, rarely ascending into the inflorescence, initially covered by the epidermis of the plants, which soon frays. Spore mass dark brown to almost black, powdery. Infection systemic, infected plants usually sterile. Spores globose to ovoid, (9.5-) 10.0-(av. 10.9) -11.5(-13.5) x (7.5-) 8.5-(av. 9.3)-10.0(-11.5) $\mu \mathrm{m}$, length/breadth ratio 1.04-(av. 1.24)-1.5, olive-brown, finely echinulate (Table 3 , Figs 3-4).

Confirmed hosts: Agrostis rupestris and A. stolonifera.

Fig. 5. Sori and spores of Ustilago jagei (A-B), U. denotarisii (C-D), U. neocopinata (E-F), U. salweyi (G-H), U. kummeri (I-J), and U. serpens s. str. (K-L). A. Sori of U. jagei on Agrostis stolonifera (GLM-F047379); B. Teliospores seen by LM; C. Sori of U. denotarisii on Arrhenatherum elatius (GLM-F105836); D. Teliospores seen by LM; E. Sori of U. neocopinata on Dactylis glomerata (GLM-F107413); F. Teliospores seen by LM; G. Sori of U. salweyi on Holcus lanatus (GLM-F107417); H. Teliospores seen by LM; I. Sori of U. kummeri on Bromus inermis (GLM- F107435); J. Teliospores seen by LM; K. Sori of U. serpens s. str. on Elymus repens (GLM-F105827); and L. Teliospores seen by LM. 
Table 2. Diagnostic bases within the Ustilago striformis and the Ustilago serpens complexes.

\begin{tabular}{|c|c|c|c|c|c|c|c|c|c|c|c|c|c|c|c|c|c|c|}
\hline \multirow[b]{3}{*}{ U. striiformis-complex } & \multicolumn{18}{|c|}{ Gen Loci } \\
\hline & \multicolumn{2}{|c|}{ atp2 } & \multicolumn{2}{|c|}{ map } & \multicolumn{2}{|c|}{ ssc1 } & \multicolumn{2}{|c|}{ myosin } & \multicolumn{2}{|c|}{$r p / 4 A$} & \multicolumn{2}{|c|}{$r p / 3$} & \multicolumn{2}{|c|}{ sdh1 } & \multicolumn{2}{|c|}{ tif2 } & \multicolumn{2}{|c|}{ ITS } \\
\hline & Pos. & Base & Pos. & Base & Pos. & Base & Pos. & Base & Pos. & Base & Pos. & Base & Pos. & Base & Pos. & Base & Pos. & Base \\
\hline on Agrostis gigantea & $x$ & $x$ & $x$ & $x$ & $x$ & $x$ & $x$ & $x$ & $x$ & $x$ & $x$ & $x$ & 138 & $\mathrm{C} / \mathrm{T}$ & $x$ & $x$ & $x$ & $x$ \\
\hline $\begin{array}{l}\text { on Agrostis stolonifera and } A . \\
\text { rupestris }\end{array}$ & 466 & $A / G$ & $x$ & $\mathrm{x}$ & $\mathrm{x}$ & $x$ & $x$ & $x$ & $\mathrm{x}$ & $\mathrm{x}$ & 92 & $A / G$ & $\mathrm{x}$ & $x$ & $\mathrm{x}$ & $x$ & $x$ & $x$ \\
\hline on Alopecurus pratensis & 358 & $A / G$ & 192 & $\mathrm{G} / \mathrm{T}$ & $x$ & $\mathrm{x}$ & 83 & $\mathrm{~T} / \mathrm{C}$ & $x$ & $x$ & $x$ & $x$ & $x$ & $x$ & $x$ & $x$ & $x$ & $x$ \\
\hline on Arrhenatherum elatius & 346 & $A / G$ & $x$ & $x$ & 182 & $\mathrm{~A} / \mathrm{C}$ & $x$ & $x$ & $x$ & $x$ & $x$ & $x$ & $x$ & $x$ & $x$ & $x$ & $x$ & $x$ \\
\hline on Bromus inermis & 191,244 & $\mathrm{G} / \mathrm{A}$ & $\mathrm{x}$ & $\mathrm{x}$ & 232 & $\mathrm{C} / \mathrm{T}$ & $x$ & $x$ & $\begin{array}{l}228,311 \\
292\end{array}$ & $\begin{array}{l}\mathrm{A} / \mathrm{G} \\
\mathrm{C} / \mathrm{T}\end{array}$ & $x$ & $x$ & $\mathrm{x}$ & $\mathrm{x}$ & 23 & $A / G$ & 621 & $\mathrm{C} / \mathrm{T}$ \\
\hline on Calamagrostis epigejos & 91 & $A / G$ & $\mathrm{x}$ & $\mathrm{x}$ & $x$ & $x$ & $\mathrm{x}$ & $x$ & $\mathrm{x}$ & $x$ & $x$ & $x$ & $x$ & $x$ & 65 & $\mathrm{~T} / \mathrm{C}$ & 102 & $\mathrm{~T} / \mathrm{C}$ \\
\hline on Calamagrostis villosa & 535 & $\mathrm{~T} / \mathrm{C}$ & $x$ & $x$ & $x$ & $x$ & $x$ & $x$ & $x$ & $x$ & $x$ & $x$ & $x$ & $x$ & $x$ & $x$ & $x$ & $x$ \\
\hline on Dactylis glomerata & $x$ & $x$ & $x$ & $x$ & 69,198 & $A / G$ & $x$ & $\mathrm{x}$ & 120 & $\mathrm{~T} / \mathrm{C}$ & 40 & $A / G$ & $x$ & $\mathrm{x}$ & $x$ & $x$ & 617 & $A / G$ \\
\hline on Deschampsia caespitosa & 22,94 & $A / G$ & 227 & $\mathrm{~T} / \mathrm{C}$ & $x$ & $x$ & 133 & $A / G$ & $x$ & $\mathrm{x}$ & 199 & $\mathrm{~T} / \mathrm{C}$ & $\mathrm{x}$ & $\mathrm{x}$ & $\mathrm{x}$ & $\mathrm{x}$ & 576 & $A / G$ \\
\hline on Festuca spp. and Lolium spp. & $\mathrm{x}$ & $\mathrm{x}$ & $\mathrm{x}$ & $\mathrm{x}$ & $\begin{array}{l}210, \\
214,231 \\
243\end{array}$ & $\begin{array}{l}A / G \\
T / C\end{array}$ & $\mathrm{x}$ & $\mathrm{x}$ & $\mathrm{x}$ & $\mathrm{x}$ & $\mathrm{x}$ & $\mathrm{x}$ & $\mathrm{x}$ & $\mathrm{x}$ & $\mathrm{x}$ & $\mathrm{x}$ & $\mathrm{x}$ & $\mathrm{x}$ \\
\hline on Holcus spp. & $x$ & $\mathrm{x}$ & $x$ & $x$ & $x$ & $x$ & $x$ & $x$ & 85 & $\mathrm{~T} / \mathrm{C}$ & 133 & $\mathrm{~T} / \mathrm{C}$ & $x$ & $x$ & $x$ & $\mathrm{x}$ & 103 & $A / G$ \\
\hline on Milium effusum & 301 & $A / G$ & $x$ & $\mathrm{x}$ & $x$ & $x$ & $x$ & $x$ & $x$ & $x$ & $\mathrm{x}$ & $x$ & $x$ & $x$ & $x$ & $x$ & 206 & $\mathrm{~T} / \mathrm{C}$ \\
\hline U. serpens-complex & Pos. & Base & Pos. & Base & Pos. & Base & Pos. & Base & Pos. & Base & Pos. & Base & Pos. & Base & Pos. & Base & Pos. & Base \\
\hline on Bromus inermis & $\mathrm{x}$ & $\mathrm{x}$ & $x$ & $\mathrm{x}$ & $\mathrm{x}$ & $x$ & $\mathrm{x}$ & $\mathrm{x}$ & $\mathrm{x}$ & $\mathrm{x}$ & $\mathrm{x}$ & $\mathrm{x}$ & $\mathrm{x}$ & $\mathrm{x}$ & $\mathrm{x}$ & $\mathrm{x}$ & $\begin{array}{l}260 \\
629\end{array}$ & $\begin{array}{l}C / T \\
G / A\end{array}$ \\
\hline on Calamagrostis epigejos & $\begin{array}{l}175 \\
181, \\
429,496\end{array}$ & $\begin{array}{l}\mathrm{G} / \mathrm{A} \\
\mathrm{T} / \mathrm{C}\end{array}$ & $\begin{array}{l}92 \\
203\end{array}$ & $\begin{array}{l}\mathrm{A} / \mathrm{C} \\
\mathrm{C} / \mathrm{T}\end{array}$ & $\begin{array}{l}88,99 \\
93\end{array}$ & $\begin{array}{l}C / G \\
G / A\end{array}$ & $\begin{array}{l}70 \\
225\end{array}$ & $\begin{array}{l}\mathrm{A} / \mathrm{G} \\
\mathrm{T} / \mathrm{C}\end{array}$ & $\begin{array}{l}7 \\
91\end{array}$ & $\begin{array}{l}C / G \\
T / C\end{array}$ & 28 & $\mathrm{G} / \mathrm{T}$ & $\begin{array}{l}13 \\
25\end{array}$ & $\begin{array}{l}\text { G / A } \\
G / T\end{array}$ & $\mathrm{x}$ & $\mathrm{x}$ & $\begin{array}{l}215 \\
522\end{array}$ & $\begin{array}{l}\mathrm{C} / \mathrm{T} \\
\mathrm{T} /-\end{array}$ \\
\hline & 352 & $A / G$ & & & 255 & $\mathrm{G} / \mathrm{T}$ & & & 187 & $\mathrm{G} / \mathrm{T}$ & & & 100 & $A / G$ & & & $\begin{array}{l}597 \\
606\end{array}$ & $\begin{array}{l}A / T \\
A / G\end{array}$ \\
\hline on Elymus repens & $\mathrm{x}$ & $x$ & $\mathrm{x}$ & $x$ & $\mathrm{x}$ & $x$ & 93 & $A / G$ & 232 & $\mathrm{~T} / \mathrm{C}$ & $\mathrm{x}$ & $x$ & $\mathrm{x}$ & $\mathrm{x}$ & $\mathrm{x}$ & $\mathrm{x}$ & $\begin{array}{l}157 \\
170\end{array}$ & $\begin{array}{l}A / G \\
C / T\end{array}$ \\
\hline on Phalaris arundinacea & 85 & $\mathrm{G} / \mathrm{A}$ & 208 & $\mathrm{~A} / \mathrm{G}$ & $x$ & $\mathrm{x}$ & $\begin{array}{l}141 \\
156\end{array}$ & $\begin{array}{l}C / A \\
T / C\end{array}$ & $\mathrm{x}$ & $\mathrm{x}$ & $\begin{array}{l}91 \\
146\end{array}$ & $\begin{array}{l}T / C \\
A / G\end{array}$ & $\begin{array}{l}58 \\
256\end{array}$ & $A / G$ & $x$ & $\mathrm{x}$ & $\begin{array}{l}19 \\
38 \\
186,596 \\
188,604\end{array}$ & $\begin{array}{l}C / A \\
C / T \\
A /- \\
G / A\end{array}$ \\
\hline
\end{tabular}

Slash $(/)=$ instead of, $\mathrm{x}=$ no diagnostic bases. 
CTTVGAGTCVAGRGGGTCBA CRGCRGGGTARATACCRAGC TCGGCRATACCACGVGAHAR BACRGTGGTGGCGTCCAAGT GKGCRAAGGTRGTRGCGGGR GCAGGRTCMGTCAAGTCATC GGCGGGCACRTARACGGCCT GBACSGARGTRATGGARCCC TTCTTGGTGGTRGTRATKCG YTCCTGCATGRSACCCATGT CRGTRGCDAGGGTRGGCTGR TAWCCKACRGCBGARGGRAT ACGRCCRAGMARRGCSGAGG TCTCGGAACCRGCYTGGGTG AARCGGAAAATRTTGTCRAT RAARAGAAGCACRTCCTGDC CCTCYTCGTCACGGAAGTAC TCRGCRATGGTRAKACCVGT VAGRGCRACRCGGGCRCGGG CWCCRGGRGGCTCGTTCATC TGACCGAAVACRAGVGCKAC CTTGGAKTYRCCBTYVAGGT TGATRACACCRGTCTCRATC ATYTCRTGGTARAGATCRTT RCCCTCACGRGTDCGYTCAC CGACACCRGTGAARACVGAG TAACCACCGTRRGCYTTRGC RACGTTGTTRATSAGYTCYT GRATRAGYACVGTCTTDCCG ACMCCRGCRCCACCR

\section{ITS $643 \mathrm{bp}$}

HCGTYGCGTCSRGCTYHNNV MYYNNNYBHYHNHBNYCYCC GAAGYYYYGAYANNKTATCA AAACCCGGCAGGNWVGMNNN NNNNVRGVRNNVRARANNNN WBRAGCTTTCGTCCGTCNTY KCNNCYDTHVAANNNNNNNR CTMATSYWTTTCRAGRRAGC CAYGRYDMAYGGCNNNNNNN NNNNNNNVVMYCCTCAVTAC CRAKCYRYCVDCYYYYHNNN NNNNNNNNNNNNNNNNNNNNN NRARRRRRNNNNBBKNCGYY CGAAACDATTCGCGGCCCTC AAACMGGCATGCTCCCCAGA TTARATCTGCMGGGAGCGCA AGGTGCGTTCAAAGATTCGA TGATTCRCTTCTGCAATTCA CATTACTTATCGCAATTCGC TGCGTTCTTCATCGAKGGGA RAACCAAGAGATCCGTTGCC AAAAGTTVNTTTKNNNNNWW TTAGACGACCGYATTACCAG YCGNNNNNNNNNNNNNNNAW

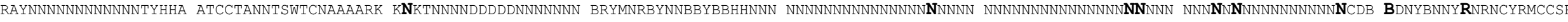
TGC

\section{SSC1 $264 \mathrm{bp}$}

CCRTCVGCRGCRGTVGARAA SACYTGCGACTTCTTGGTDG GGATVGTGGTRTTRCGGTTR ATVAGRCGVGTGAAVACACC RCCVAGNGTCTGRATACCSA GVGARAGVGGVGTRACRTCG AGSARVARAAYRTCGGTRAC CTGRCCGGAVAGVACACCAC CCTGRATSGARGCRCCRATR GMVACRGCCTCRTCKGGRTT RACRCCCTTRCTNRGGTCRC GCTTGAARATRYYCTTGACV GTYTCRAGVACCTTKGGCAT DCGG

\section{map $251 \mathrm{bp}$}

TYCGHKCCGARATCAARGCN CACMTCAARAATGTBCAGCA RGAYACBGGCAARCTYGCCA ACTCGGTYGMMAAGGAGCGY GARATGTCBACMAARWYGAT YACCGARCTYGCYCGHTCCA TCACYYTSCTCAARAAYACB CCYATGAGYGTCACBGCBCG HGARGAYCCYTACGCYGCCA AYCARBMSGTCKWYCGYCAR CTY YAGCRCCAGGTCAAYGA GGARAAYGCYCTBCARAART CSATYATCATC

\section{myosin $257 \mathrm{bp}$}

CTCTTRTTCTGRTTDGGCTT GATKGTDCKRATRTAVGAHG GCTGNGCNYKCATVARVTTY TCNACHARVRCRTTKGCRCT NGHYTTKATACGRTCRCCVG CRGTRGGVGGVCKCTTYTTR YTRTYRGGRTYNRGDCGDTC NGGRAARAGYTTYTGVARRT ASRAGTTDKBRCTYGARTCR ACVARRTCVARRATRTCYTT RAGVAGYGMRTCYTTRTTBT TRTYBGTCATDCCYTSRACR TTGTACATGACRTCRCC

\section{$\underline{r P 14 \mathrm{~A} 415 \mathrm{bp}}$}

SCTNKMNGARRTHCCBYTSG TYRTBKCBGAYSMBGCYGAG RRYYTSACSAARACCAAGGA GGCYRTYRCBCTBCTCAAGK CBVTYAAYGCYTWCRMSGAY GTYRYYAAGGTSTCSAACTY GCGCAAGRTYCGYGCNGGYG TKGGYAAGMTSCGCAACCGY CGCCAYACSCAGCGHCGYGG TCCYCTKGTBATYTACAACM AGGAYGCCGGYATYGTYAAG GCBTTCCRYAAHGTBCCYGG YGTKGAGCTSTGCTCNGYYG ASCGYCTSAACMTSCTCCAG CTYGCYCCYGGHGGACAYMT BGGYCGHTTYRTCATYTTCA CBSAGTCYGCYTTYGGCCGC CTCGACRAGGTSTTYGCYGC CAAGWMBRGYTTCAYBCTNC CYAAGGCYAAGATYGCYAAC ACBGAYGTBACSCGC

$\underline{\operatorname{rp} 13218 \mathrm{bp}}$

CANYGGYGCYTSSATCTSBC GHCAGCTBGAGCGCATCAR AAGTACTGCACYGTBGTBCG TSTBCTYGCYCACACYCAGR TBCGCAAGACBRGYC GACTTYGCSAAGGAGCACTT YGAGAAGACBTTYGMBGTY

\section{sdh1 269bp}

RTCYTGVAGRGRAGVCCRG CWCGBGARACCATRGCCATR CCRTCACCVGTGCAVGTRTG VGCVGARGTGGCVGAGAAGT ARGCACGDCCRTADCCRCCD GTGGCVAGVACRGTCTTRTG RGCRCGGAADCGGTGRAYKG TRCCRTCCTCCATRTTRAGD GCRGYVACACCGACRCAYTC GCCRTCYTCCATGATVARGT CRAGVGCRAARTACTCGATR AARAAGTTKGTGTTRTGKCG RAGCGACTGDCCRTAVAGSG TGTGMAGCA

\section{tif2 $244 \mathrm{bp}$}

TGTCGTARACACGRCCRGGV GTRCCRACRACGACCTGRGC RCCBTCRTTGAGCTTRGCCA TGTCYTCRCGRACGTTRGTR CCRCCRATGCARGCGTGGCA GTYRAYCTTCATGTAGTCRC CRAGNGCRATRACGACCTTY TGRATCTGCTGRGCVAGCTC RCGRGTRGGVGCRAGGATVA GVGCYTGRACVGCCTKGATS YYRGGGTCRATDCGCTGVAR RATGGCRATSGAGAAGGTRG CBGT

Fig. 6. Alignment consensus sequences for the alignments used in this study with positions of diagnostic bases highlighted in bold face. 
Confirmed distribution: Germany and Switzerland.

Notes: It seems possible that $U$. jagei on Agrostis stolonifera s. lat. represents a species complex, and further investigations with more specimens and additional gene loci are needed to clarify this situation.

\section{Ustilago kummeri J. Kruse \& Thines, sp. nov. MycoBank MB819628}

(Fig. 5I-J)

Etymology: Named after the mycologist Volker Kummer from Potsdam (Germany), who has made significant contributions to the knowledge of phytopathogenic fungi and has enabled well-sampled phylogenetic investigations in various plant pathogens by his outstanding ability to recognise easily overlooked plant pathogens.

Diagnosis: Differs from species of the $U$. striiformis species complex in the larger spores and taller warts. Furthermore, $U$. kummeri shares one sequence motif at positions 20-27 (AACCCAAC) with other coarsely ornamented stripe smuts, and many SNPs distinguishing it from species of the $U$. striiformis species complex. Within the $U$. serpens-complex, $U$. kummeri can be distinguished from other species based on the host-specific occurrence on Bromus inermis. Furthermore, U. kummeri differs in two diagnostic bases from $U$. serpens on Elymus repens - in the ITS region there is an $C$ instead of a $G$ at position 260 and $G$ instead of an $A$ at position 629 (Table 2, Fig. 6).

Type: Germany: Brandenburg: Middlemark, Uetz: Hinterer Werder, southwest corner between Sacrow-ParetzerChannel und Havel-Channel, on Bromus inermis, 19 June 2010, V. Kummer (GLM-F107435 - holotype; VK 2577/17 isotype).

Description: Sori as long, narrow streaks parallel to vascular bundles, mostly in the leaves, rarely ascending to the inflorescence, initially covered by the epidermis of the plants, which soon frays. Spore mass dark brown, powdery. Infection systemic, infected plants mostly sterile. Spores ovoid to globose, (11.0-) 12.0- (av. 13.0) -14.0 (-15.5) × (9.0-) 10.5(av. 11.5) -12.0 (-13.5), length/breadth ratio 1.04- (av. 1.15) -1.41 , olive-brown, coarsely verrucose to echinulate (Table 3 , Figs 5-6).

\section{Confirmed host: Bromus inermis.}

\section{Confirmed distribution: Germany.}

Notes: It seems likely that additional species will be discovered in the $U$. serpens clade once more stripe-smuts with coarse spore ornamentation will be scrutinised.

Ustilago Ioliicola Ciferri, Fl. Ital. Crypt., Par. I. Fungi, Fasc. 17: 345 (1938).

Type: Germany: Berlin: Berlin-Weissensee, on Lolium perenne, Sept. 1877, E. Ule [Rabenhorst, Fungi Eur. no.
2491] (FR - lectotype designated here, MBT 380633; from one of the several duplicate collections treated as "lectotype" by Lindeberg, Symb. Bot. Upsal. 16 (2): 136, 1959).

Confirmed hosts: Festuca arundinacea s. lat. and Lolium perenne.

\section{Confirmed distribution: Germany.}

Notes: Within the $U$. striiformis species complex, $U$. Ioliicola can be distinguished from other species based on the specific occurrence on the closely related hosts Festuca arundinacea s. lat. and Lolium perenne. Furthermore, U. Ioliicola differs in four diagnostic bases from all other species within the $U$. striiformis species complex included in this study - in the ssc 1 locus there is an A instead of a $G$ at positions 210, 214 and 231, and a T instead of a C at position 243 (Table 2, Fig. 6).

Ustilago milii (Fuckel) Liro, Ann. Acad. Sci. Fenn., ser. A 17 (1): 78 (1924).

Basionym: Tilletia milii Fuckel, Jb. nassau. Ver. Naturk. 2324: 40 (1870).

Type: Germany: Hesse: Rabenkopf Mt., near Oestrich, on Milium effusum, L. Fuckel [Fungi Rhenani no. 2410] (FR lectotype designated here, MBT 380634, from one of the several duplicate collections treated as "lectotype" in Fuckel, Fungi Rhenani no. 2410).

Confirmed host: Milium effusum.

Confirmed distribution: Germany.

Notes: Within the $U$. striiformis species complex, $U$. milii can be distinguished from other species based on the hostspecific occurrence on Milium effusum. Furthermore, U. milii differs in two diagnostic bases from all other species within the $U$. striiformis species complex included in this study - in the atp2 gene there is an A instead of a $G$ at position 301, and in the ITS there is a T instead of a $\mathrm{C}$ at position 206 (Table 2, Fig. 6).

\section{Ustilago neocopinata J. Kruse \& Thines, sp. nov. MycoBank MB819630}

(Fig. 5E-F)

Etymology: Highlights the unexpected finding that there are several distinct and host-specific species within the $U$. striiformis species complex.

Diagnosis: Within the $U$. striiformis species complex, $U$. neocopinata can be distinguished from other species based on the host-specific occurrence on Dactylis glomerata. Furthermore, $U$. neocopinata differs in five diagnostic bases from all other species within the $U$. striiformis species complex included in this study - in the ssc1 gene there is an A instead of a $G$ at positions 69 and 198, in the rp/4A gene there is a $T$ instead of a $C$ at position 120 , in the $r p / 3$ gene there is an $A$ instead of a $G$ at position 40 , and in the ITS region there is an A instead of a $\mathrm{G}$ at position 617 (Table 2, Figs 5-6). 
Table 3. Measurements from 100 teliospores for four different species of the Ustilago striformis-complex on Agrostis stolonifera, Dactylis glomerata, Arrhenatherum elatius, and Holcus lanatus, as well as two species of the Ustilago serpens-complex on Elymus repens and Bromus inermis.

\section{Ustilago striiformis-complex}

U. jagei sp. nov.

U. denotarisii

U. neocopinata sp. nov. U. salveii

Ustilago serpens-complex

on Agrostis stolonifera on Arrhenatherum elatius on Dactylis glomerata

on Holcus lanatus spores spores

\section{$U$. serpens}

U. kummeri sp. nov. spores

spores

No. length width $\mathrm{l} / \mathrm{b}$ length width $\mathrm{l} / \mathrm{b} \quad$ length width $\mathrm{l} / \mathrm{b}$ length width $\mathrm{l} / \mathrm{b} \quad$ length width $\mathrm{l} / \mathrm{b} \quad$ length width $\mathrm{l} / \mathrm{b}$, spores

spores

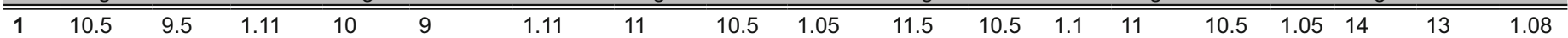

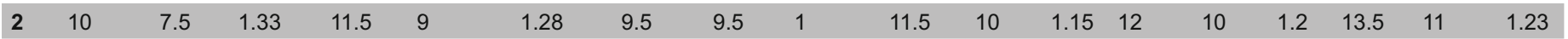

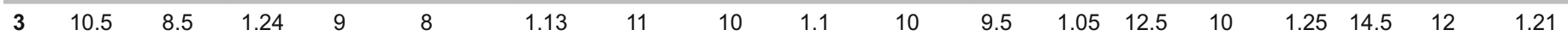

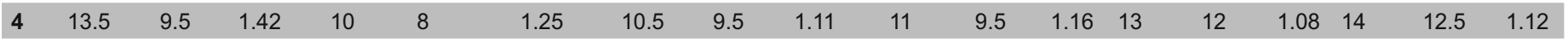

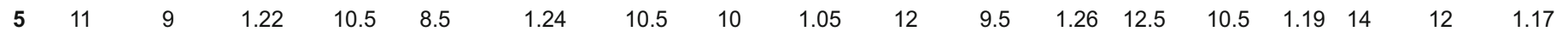

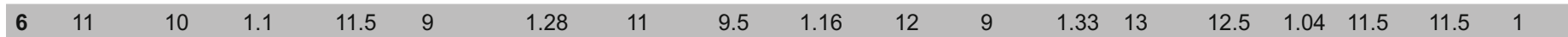

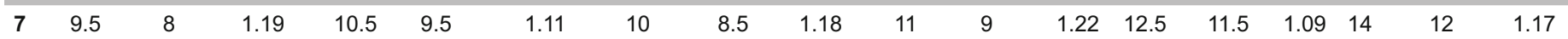

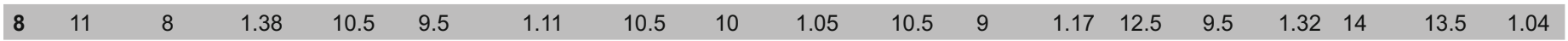

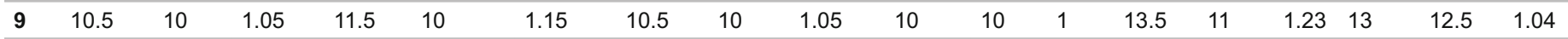

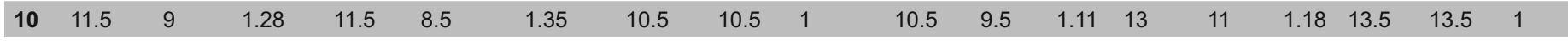

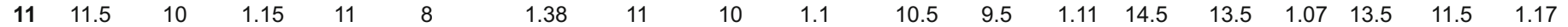

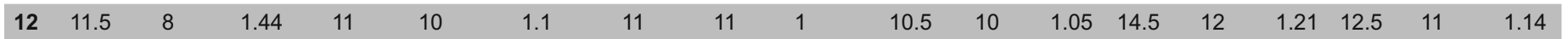

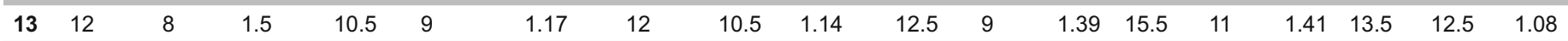

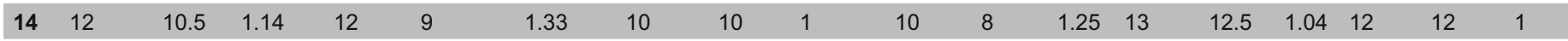

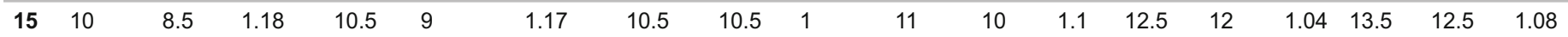

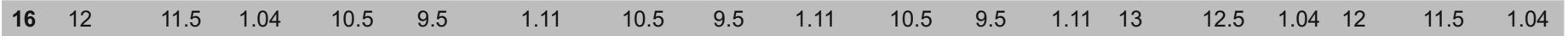

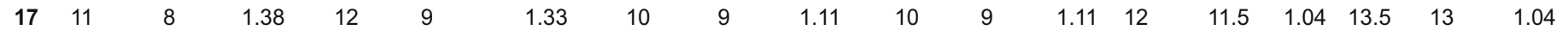

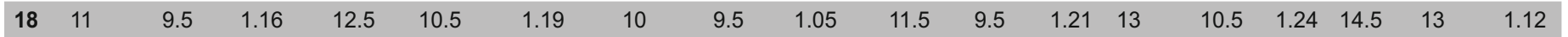

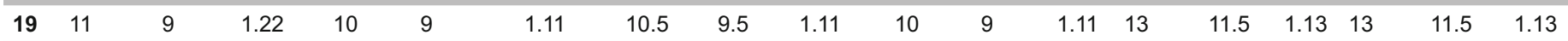

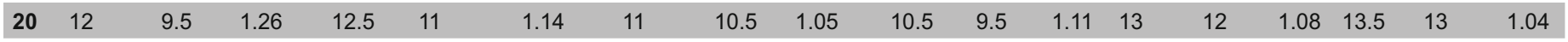

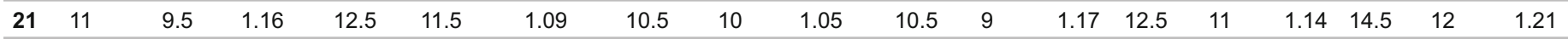

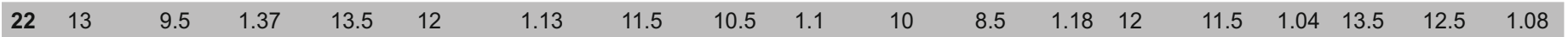

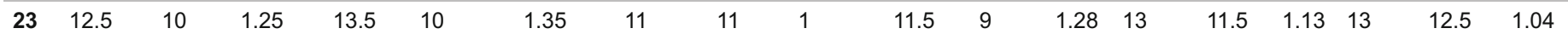

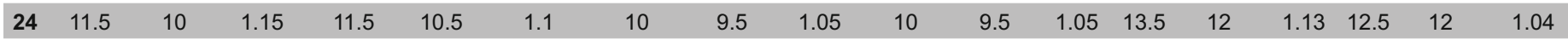

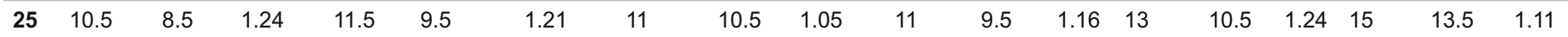

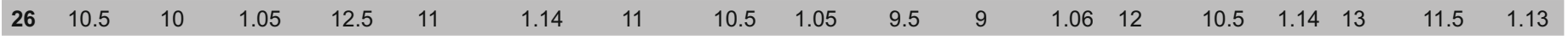

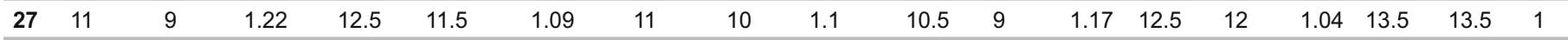

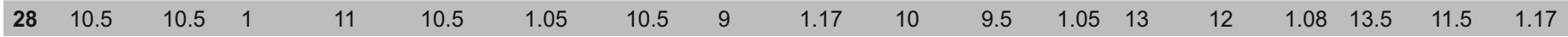

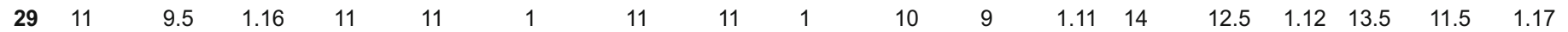

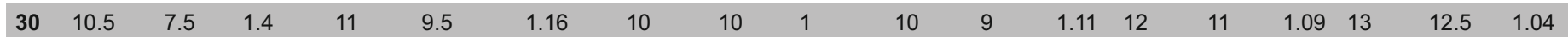

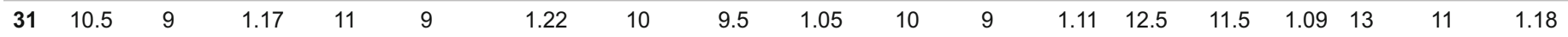

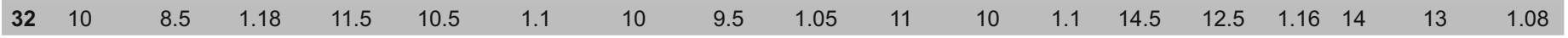

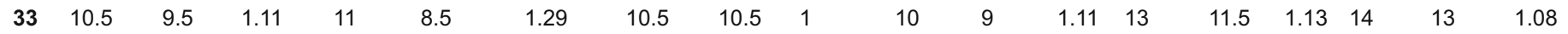

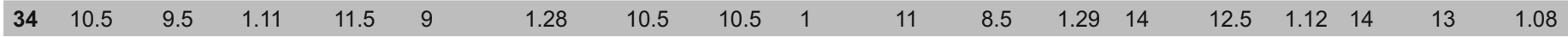

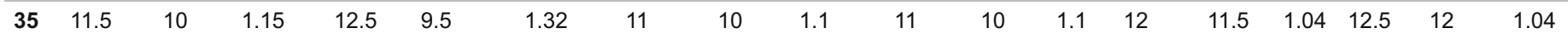

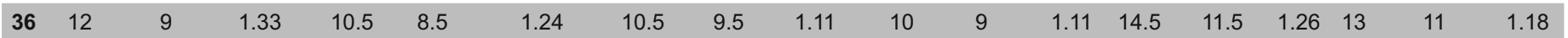

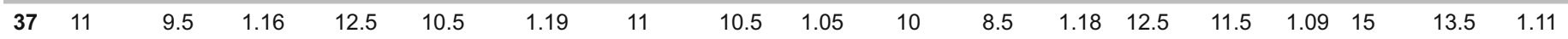

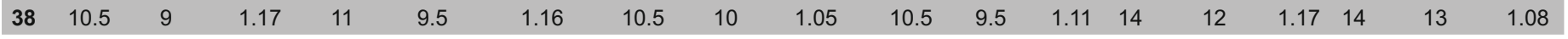

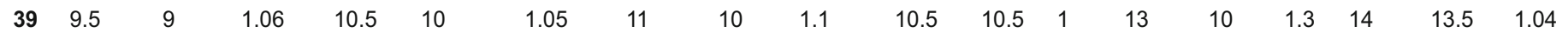

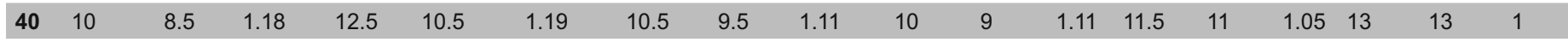

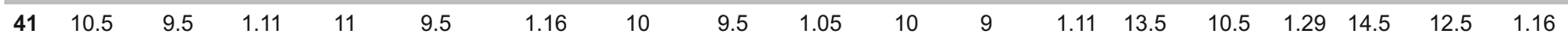

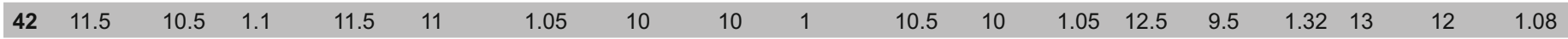

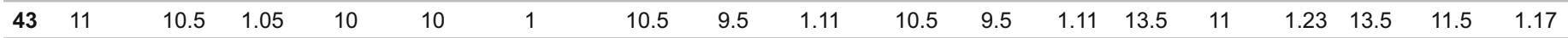

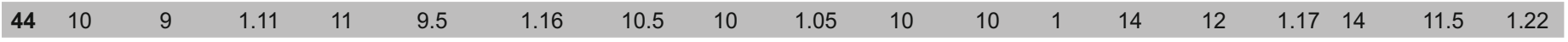

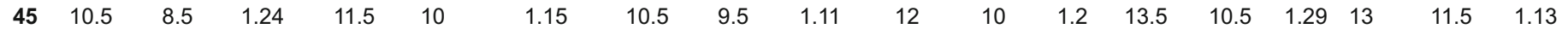

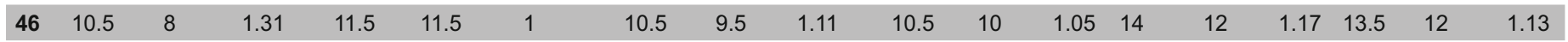

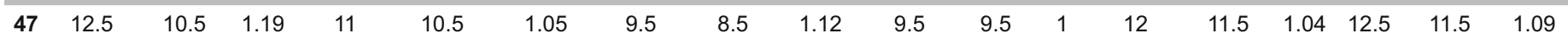

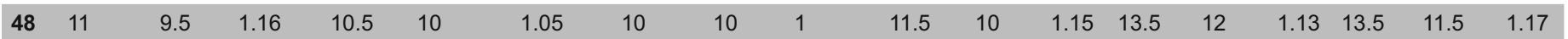

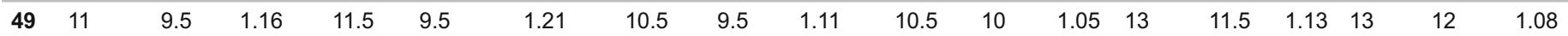

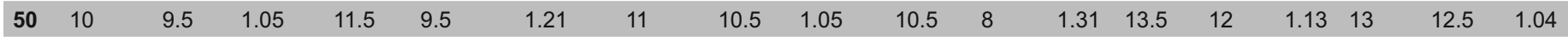

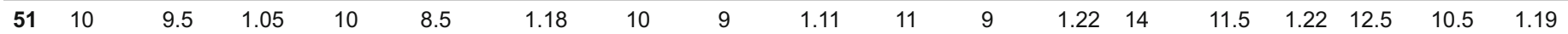

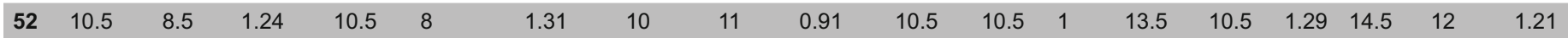


Table 3. (Continued).

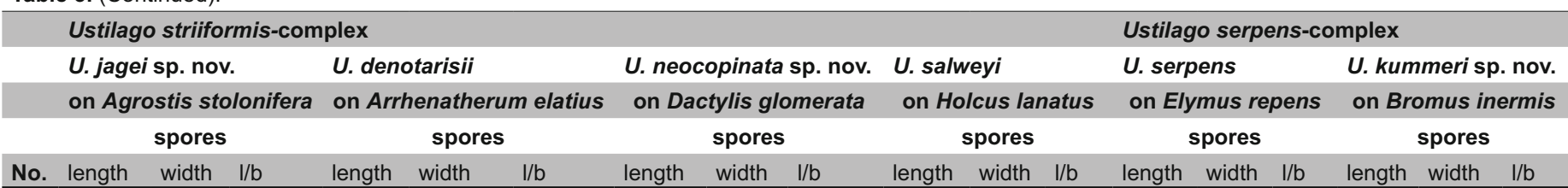

\begin{tabular}{|c|c|c|c|c|c|c|c|c|c|c|c|c|c|c|c|c|c|c|}
\hline 53 & 12 & 9.5 & 1.26 & 10 & 8.5 & 1.18 & 9 & 9 & 1 & 11.5 & 10 & 1.15 & 14 & 11.5 & 1.22 & 14 & 12 & 1.17 \\
\hline 54 & 10.5 & 10.5 & 1 & 11 & 8.5 & 1.29 & 10 & 9.5 & 1.05 & 10.5 & 9 & 1.17 & 13 & 12 & 1.08 & 13.5 & 12 & 1.13 \\
\hline 55 & 10.5 & 10.5 & 1 & 12 & 9.5 & 1.26 & 10 & 9.5 & 1.05 & 10 & 10 & 1 & 12 & 11 & 1.09 & 14 & 12 & 1.17 \\
\hline 56 & 11 & 10 & 1.1 & 9.5 & 9 & 1.06 & 9.5 & 9.5 & 1 & 10.5 & 10.5 & 1 & 12 & 12 & 1 & 13 & 13 & 1 \\
\hline 57 & 10.5 & 9 & 1.17 & 10 & 8.5 & 1.18 & 11.5 & 10.5 & 1.1 & 11 & 9.5 & 1.16 & 13 & 10.5 & 1.24 & 14 & 13 & 1.08 \\
\hline 58 & 10 & 10 & 1 & 11.5 & 9.5 & 1.21 & 10 & 9 & 1.11 & 10.5 & 10 & 1.05 & 14.5 & 10.5 & 1.38 & 13 & 12.5 & 1.04 \\
\hline 59 & 11 & 10 & 1.1 & 11 & 10 & 1.1 & 10 & 7.5 & 1.33 & 10.5 & 9.5 & 1.11 & 13 & 11.5 & 1.13 & 13.5 & 12 & 1.13 \\
\hline 60 & 10.5 & 10.5 & 1 & 12 & 9.5 & 1.26 & 10 & 10 & 1 & 10 & 9.5 & 1.05 & 13 & 12 & 1.08 & 14.5 & 12 & 1.21 \\
\hline 61 & 10.5 & 8.5 & 1.24 & 11 & 10 & 1.1 & 10.5 & 10 & 1.05 & 10.5 & 9.5 & 1.11 & 13.5 & 10 & 1.35 & 14.5 & 13 & 1.12 \\
\hline 62 & 11.5 & 9 & 1.28 & 10.5 & 10 & 1.05 & 11 & 9 & 1.22 & 10.5 & 10 & 1.05 & 13 & 11.5 & 1.13 & 14.5 & 13 & 1.12 \\
\hline 63 & 10.5 & 8.5 & 1.24 & 10.5 & 9.5 & 1.11 & 10.5 & 9.5 & 1.11 & 9.5 & 9.5 & 1 & 12.5 & 12 & 1.04 & 13.5 & 12 & 1.13 \\
\hline 64 & 10.5 & 9.5 & 1.11 & 10.5 & 10 & 1.05 & 11 & 10.5 & 1.05 & 10 & 9.5 & 1.05 & 12.5 & 12 & 1.04 & 14 & 12.5 & 1.12 \\
\hline 65 & 10 & 10 & 1 & 10 & 8.5 & 1.18 & 10.5 & 10.5 & 1 & 11 & 9 & 1.22 & 14.5 & 10.5 & 1.38 & 13 & 12.5 & 1.04 \\
\hline 66 & 10.5 & 8.5 & 1.24 & 11.5 & 11 & 1.05 & 11 & 11 & 1 & 10.5 & 8.5 & 1.24 & 11.5 & 10.5 & 1.1 & 13 & 12.5 & 1.04 \\
\hline 67 & 11 & 10.5 & 1.05 & 11 & 9.5 & 1.16 & 11.5 & 10 & 1.15 & 11 & 9.5 & 1.16 & 15 & 12 & 1.25 & 13.5 & 12.5 & 1.08 \\
\hline 68 & 10.5 & 8.5 & 1.24 & 11 & 9.5 & 1.16 & 11 & 11 & 1 & 10.5 & 9.5 & 1.11 & 12.5 & 11 & 1.14 & 14 & 13 & 1.08 \\
\hline 69 & 10 & 10 & 1 & 11.5 & 10.5 & 1.1 & 11.5 & 11 & 1.05 & 10 & 9 & 1.11 & 14 & 11 & 1.27 & 14.5 & 14 & 1.04 \\
\hline 70 & 10 & 10 & 1 & 11.5 & 11 & 1.05 & 10 & 9.5 & 1.05 & 11 & 10 & 1.1 & 12 & 11 & 1.09 & 13.5 & 12.5 & 1.08 \\
\hline 71 & 11 & 9 & 1.22 & 11 & 10 & 1.1 & 9 & 9 & 1 & 10.5 & 9 & 1.17 & 13 & 10.5 & 1.24 & 13 & 12.5 & 1.04 \\
\hline 72 & 10 & 10 & 1 & 10.5 & 10 & 1.05 & 10 & 9.5 & 1.05 & 11 & 10.5 & 1.05 & 13 & 12 & 1.08 & 13.5 & 12.5 & 1.08 \\
\hline 73 & 10 & 10 & 1 & 13 & 10 & 1.3 & 11 & 10 & 1.1 & 9.5 & 9 & 1.06 & 11.5 & 11.5 & 1 & 13.5 & 13 & 1.04 \\
\hline 74 & 10.5 & 8 & 1.31 & 11 & 9 & 1.22 & 10.5 & 9.5 & 1.11 & 11 & 9.5 & 1.16 & 14 & 11 & 1.27 & 13.5 & 13 & 1.04 \\
\hline 75 & 10 & 9.5 & 1.05 & 11.5 & 10 & 1.15 & 10.5 & 9.5 & 1.11 & 10.5 & 10.5 & 1 & 12 & 10.5 & 1.14 & 15.5 & 13.5 & 1.15 \\
\hline 76 & 11.5 & 9 & 1.28 & 12 & 10.5 & 1.14 & 11 & 9.5 & 1.16 & 10.5 & 10 & 1.05 & 11.5 & 9 & 1.28 & 13 & 12.5 & 1.04 \\
\hline 77 & 11 & 10 & 1.1 & 10.5 & 10 & 1.05 & 9 & 9 & 1 & 11.5 & 9.5 & 1.21 & 12.5 & 11 & 1.14 & 14 & 12.5 & 1.12 \\
\hline 78 & 11.5 & 9.5 & 1.21 & 10.5 & 10 & 1.05 & 9.5 & 9.5 & 1 & 9.5 & 8.5 & 1.12 & 11 & 10.5 & 1.05 & 14.5 & 12 & 1.21 \\
\hline 79 & 11 & 9 & 1.22 & 11.5 & 8.5 & 1.35 & 11 & 10 & 1.1 & 10 & 9 & 1.11 & 13 & 11 & 1.18 & 13.5 & 12 & 1.13 \\
\hline 80 & 11.5 & 9.5 & 1.21 & 10.5 & 9.5 & 1.11 & 10 & 8.5 & 1.18 & 11 & 8.5 & 1.29 & 11.5 & 11 & 1.05 & 13.5 & 12.5 & 1.08 \\
\hline 81 & 9.5 & 9.5 & 1 & 11 & 9.5 & 1.16 & 10.5 & 10 & 1.05 & 11.5 & 9 & 1.28 & 11.5 & 11 & 1.05 & 13.5 & 10.5 & 1.29 \\
\hline 82 & 11 & 9.5 & 1.16 & 10.5 & 10 & 1.05 & 11 & 9.5 & 1.16 & 10.5 & 10 & 1.05 & 12.5 & 11 & 1.14 & 13 & 13 & 1 \\
\hline 83 & 10.5 & 10.5 & 1 & 11.5 & 9 & 1.28 & 11.5 & 9.5 & 1.21 & 11 & 9.5 & 1.16 & 12.5 & 12 & 1.04 & 14.5 & 13 & 1.12 \\
\hline 84 & 11.5 & 10 & 1.15 & 11 & 9 & 1.22 & 10 & 9.5 & 1.05 & 10 & 9.5 & 1.05 & 12 & 9.5 & 1.26 & 13.5 & 12.5 & 1.08 \\
\hline 85 & 11 & 10 & 1.1 & 10 & 10 & 1 & 11 & 9.5 & 1.16 & 10 & 8 & 1.25 & 13.5 & 11 & 1.23 & 14 & 13 & 1.08 \\
\hline 86 & 11 & 9 & 1.22 & 10.5 & 9.5 & 1.11 & 11.5 & 11 & 1.05 & 9.5 & 7.5 & 1.27 & 14 & 9.5 & 1.47 & 14 & 12 & 1.17 \\
\hline 87 & 11 & 10.5 & 1.05 & 10.5 & 9 & 1.17 & 11.5 & 10.5 & 1.1 & 11 & 8.5 & 1.29 & 12.5 & 12 & 1.04 & 15 & 13.5 & 1.11 \\
\hline 88 & 11 & 9 & 1.22 & 11 & 9.5 & 1.16 & 10 & 9 & 1.11 & 10 & 9 & 1.11 & 12 & 12 & 1 & 12.5 & 12.5 & 1 \\
\hline 89 & 10 & 7.5 & 1.33 & 11.5 & 8.5 & 1.35 & 9.5 & 9 & 1.06 & 10 & 9.5 & 1.05 & 15 & 12.5 & 1.2 & 13.5 & 11.5 & 1.17 \\
\hline 90 & 11 & 9.5 & 1.16 & 10 & 9.5 & 1.05 & 11 & 9.5 & 1.16 & 11 & 10 & 1.1 & 14.5 & 12 & 1.21 & 12 & 12 & 1 \\
\hline 91 & 10.5 & 9 & 1.17 & 13.5 & 11 & 1.23 & 11 & 9.5 & 1.16 & 11 & 10.5 & 1.05 & 12 & 11.5 & 1.04 & 13.5 & 13 & 1.04 \\
\hline 92 & 10.5 & 9 & 1.17 & 13.5 & 10.5 & 1.29 & 9.5 & 9.5 & 1 & 11.5 & 10 & 1.15 & 14 & 11 & 1.27 & 13 & 12 & 1.08 \\
\hline 93 & 10 & 8.5 & 1.18 & 13 & 10.5 & 1.24 & 9.5 & 7.5 & 1.27 & 11.5 & 9.5 & 1.21 & 13.5 & 11 & 1.23 & 14 & 12.5 & 1.12 \\
\hline 94 & 11 & 9.5 & 1.16 & 11.5 & 10.5 & 1.1 & 11 & 11 & 1 & 10.5 & 9.5 & 1.11 & 13 & 11 & 1.18 & 13.5 & 11.5 & 1.17 \\
\hline 95 & 10.5 & 9 & 1.17 & 11 & 11 & 1 & 11 & 10 & 1.1 & 10.5 & 10 & 1.05 & 14.5 & 12 & 1.21 & 14.5 & 12 & 1.21 \\
\hline 96 & 10.5 & 7.5 & 1.4 & 12 & 10.5 & 1.14 & 10.5 & 8.5 & 1.24 & 10.5 & 9.5 & 1.11 & 12.5 & 10.5 & 1.19 & 14.5 & 13 & 1.12 \\
\hline 97 & 11.5 & 9.5 & 1.21 & 11 & 11 & 1 & 10.5 & 10 & 1.05 & 10.5 & 9.5 & 1.11 & 12.5 & 11.5 & 1.09 & 13 & 11 & 1.18 \\
\hline 98 & 11.5 & 9.5 & 1.21 & 11 & 9 & 1.22 & 13 & 11 & 1.18 & 11 & 10 & 1.1 & 11.5 & 11 & 1.05 & 13.5 & 11.5 & 1.17 \\
\hline 99 & 13.5 & 11.5 & 1.17 & 10.5 & 10 & 1.05 & 10 & 9 & 1.11 & 10.5 & 9.5 & 1.11 & 12.5 & 11.5 & 1.09 & 14.5 & 13.5 & 1.07 \\
\hline 100 & 10.5 & 9.5 & 1.11 & 11 & 10 & 1.1 & 10 & 10 & 1 & 11 & 9.5 & 1.16 & 13.5 & 12.5 & 1.08 & 13.5 & 11.5 & 1.17 \\
\hline
\end{tabular}


Type: Germany: Bavaria: Upper Franconia, Kronach county, Wallenfels, in the direction of the sewage treatment plant downstream of Stumpfenschneidmühle, on Dactylis glomerata, 15 July 2012, J. Kruse (GLM-F107413 - holotype).

Description: Sori as long small streaks parallel to vascular bundles, mostly in the leaves, very rarely ascending to the inflorescence, initially covered by the epidermis of the plants, which soon frays. Spore mass dark brown to almost black, powdery. Infection systemic, infected plants mostly sterile. Spores mostly globose, rarely ovoid, (9.0-) 10.0- (av. 10.5)-11.0 (-13.0) × (7.5-) 9.0- (av. 9.8) -10.5 (-11) $\mu \mathrm{m}$, length/breadth ratio 1.00- (av. 1.07) -1.18 , olive-brown, finely echinulate (Table 3, Figs 5-6).

Notes: As the host is widespread throughout the Holarctic region, it is conceivable that the species will prove to have a much wider distribution range than currently known.

Ustilago salweyi Berk. \& Broome, Ann. Mag. Nat. Hist. 5: 463 (1850).

(Fig. 5G-H)

Type: UK: Channel Islands: Guernsey, St Martin's, on Holcus lanatus [originally misidentified as Dactylis glomerata fide Hubbard, in Stevenson, Plant Dis. Rep. 30: 57, 1946], 1847, T. Salwey (K-M - holotype; K-M00022071 - isotype).

Synonyms: Uredo striiformis Westend., Bull. Acad. R. Sci. Belg., cl. sci. 18: 406 (1852); as "striaeformis".

Uredo salveii (Berk. \& Broome) Oudem., Prodromus Florae Bataviae, $2^{\text {nd }}$ edn, 4: 180 (1866).

Tilletia debaryana A.A. Fisch. Waldh., in Rabenhorst, Fungi eur. no. 1097 (1867).

Tilletia striiformis (Westend.) Magnus, Malpighia 1: 8 (1875). Ustilago striiformis (Westend.) Niessl, Hedwigia 15: 1 (1876).

Tilletia salveii (Berk. \& Broome) P. Karst., Bidrag. Kännedom. Finlands Naurt. Folk. 6: 102 (1884).

\section{Confirmed hosts: Holcus lanatus and H. mollis.}

Confirmed distribution: Belgium, Germany, and UK.

Notes: Spores globose to ovoid, standard range (9.5-)10.0(av. 10.6)-11.0 (-12.5) × (7.5-) 9.0-(av. 9.4)-10.0(-10.5) $\mu \mathrm{m}$, finely echinulate, length/breadth ratio $1.00-(a v .1 .15)-1.39$. Within the $U$. striiformis species complex, $U$. salweyi can be distinguished from other species based on the host-specific occurrence on Holcus lanatus and $H$. mollis. Furthermore, $U$. salweyi differs in three diagnostic bases from all other species within the striiformis species complex included in this study - in the rp/4A gene there is a $\mathrm{T}$ instead of a $\mathrm{C}$ at position 85 , in $r p / 3$ there is a $T$ instead of a $C$ at position 133 , and in the ITS region there is an A instead of a $G$ at positions 103 (Table 2, Fig. 6).

The original host was misidentified as Dactylis glomerata, but this was found to be incorrect and actually Holcus lanatus by the leading grass specialist C.E. Hubbard (in Stevenson 1946). David Hawksworth also studied the type materials in $\mathrm{K}-\mathrm{M}$ and concurs. Hosts in their vegetative stage can be misidentified, as some characteristics, such as leaf shape, ligula, and general habit can be modified as a consequence of infection.

Ustilago scaura Liro s. lat. , Ann. Acad. Sci. Fenn., ser. A, 17(1): 73 (1924).

Replaced name: Tilletia avenae Ule, Verh. Bot. Vereins Prov. Brandenburg 25: 214 (1884).

Type: Germany: Bavaria: Coburg, Fortress, on Avena pratensis (i.e. Helictotrichon pratense), June 1879, E. Ule (s. n. - lost); Hesse: county Tann/Rhön, at Galgenmount, on Avena pubescens [now, Helictotrichon pubescens], 16 Sept. 1990, H. Scholz (B 700014830 - neotype designated here, MBT 380637).

Non Ustilago avenae (Pers.) Rostrup, Overs. K. danske Vidensk. Selsk. Forh. Medlemmers Arbeider. 13 (1890).

Confirmed host: Helictotrichon pubescens, H. pratense?

\section{Confirmed distribution: Germany.}

Notes: Within the U. striiformis species complex, U. scaura s. lat. can be distinguished from other species based on the host-specific occurrence on Helictotrichon pratense and $H$. pubescens. Furthermore, U. scaura s. lat. differs in one diagnostic base from all other species within the $U$. salweyi species complex included in this study, except $U$. denotarisii on Arrhenatherum spp., in having a $T$ instead of a $C$ at position 628 in the ITS region, and from $U$. denotarisii on Arrhenatherum elatius in having a 13 nucleotide deletion at positions 222-241 in the ITS alignment (Table2, Fig. 6).

Since the type has been lost, we designate a neotype for Ustilago scaura with material on the closely related $H$. pubescens.

Ustilago scrobiculata Liro, Ann. Acad. Sci. Fenn., ser. A 17(1): 68 (1924).

Type: Finland: Nyland: Pornainen, Kirveskoski, on Calamagrostis arundinacea, 9 Aug. 1916, T. Putkonen \& J. I. Liro ( $\mathrm{H}-$ lectotype, designated by Lindeberg, Symb. Bot. Upsal. 16 (2): 130 (1959).

Synonym: ? Ustilago deyeuxiae L. Guo, Mycosystema 6: 51 (1993).

Reported hosts: Calamagrostis spp. (see Vánky 2012: 1265).

Reported distribution: Asia and Europe.

Notes: This species shares one sequence motif with other coarsely ornamented stripe smuts (AACCCAAC at positions 20-27), which distinguishes it from species of the Ustilago striiformis species complex, and many additional single SNPs. Within the $U$. serpens species complex, $U$. scrobiculata differs in 21 diagnostic bases from other species (Table 2, Fig. 6). It seems possible that $U$. deyeuxiae has not been sampled 
on Calamagrostis arundinacea, as the host of $U$. deyeuxiae is given as "Deyeuxia arundinacea" by Guo (1993), which is often seen as a synonym of $D$. pyramidalis in Asian literature (e.g. Shenglian et al. 2006). Thus, it seems possible that the species needs to be reconsidered as independent from $U$. scrobiculata once sequence data from the type specimen become available.

Ustilago serpens (P. Karst.) B. Lindeb., Symb. Bot. Upsal. 16(2): 133 (1959).

Basionym: Tilletia serpens P. Karst., Fungi Fenn. Exs., fasc. 6 : no. 599 (1866).

Type: Finland: Merimasku, on "Dactylis glomerata" [redetermined as Elymus repens by Lindeberg, Symb. Bot. Upsal. 16(2): 133, 1959], July 1862, P. Karsten [Fungi Fenn. Exs no. 599] (HUV 10432 - lectotype designated here; MBT 380638 from one of the several duplicate collections treated as "lectotype" by Lindeberg, Symb. Bot. Upsal. 16(2): 133, 1959).

\section{Confirmed host: Elymus repens.}

\section{Confirmed distribution: Finland and Germany.}

Notes: The spores are small to medium sized, (11.5-) 13.0(av. 13.5) -14.5 (-15.5) × (10.5-) 11.5 (av. 12.5) -13.0 (-14.0) $\mu \mathrm{m}$, with a length/breadth ratio of $1.00-($ av. 1.09)-1.23 and with coarsely verrucose ornamentation. This species shares one sequence motif with other coarsely ornamented stripe smuts (AACCCAAC at position 20-27), which distinguishes it from species of the $U$. striiformis species complex and many additional SNPs. Within the $U$. serpens-complex, $U$. serpens can be distinguished from other species based on four diagnostic bases: in the myosin gene there is an A instead of $\mathrm{G}$ at position 93, in rp/4A gene there is a $\mathrm{T}$ instead of a $\mathrm{C}$ at position 232 and in the ITS locus there is a $C$ instead of a $T$ at position 260, and a $G$ instead of an A at position 629 (Table 2, Fig. 6).

Vánky (2012) lists several additional hosts for $U$. serpens. Due to the narrow specialization of stripe-smut revealed in this study, however, it seems likely that these harbour several distinct species. Until sequence data become available for these host-pathogen combinations, Ustilago on these other hosts is probably best referred to as $U$. serpens $s$. lat.

\section{DISCUSSION}

In this study, the closely related species of the Ustilago striiformis-complex and some other leaf stripe Ustilago smuts were investigated using multigene phylogenetic reconstructions to clarify their relationships. In total, 62 specimens of the $U$. striiformis species complex (incl. U. calamagrostidis) and four other leaf stripe smuts ( $U$. echinata, $U$. filiformis, $U$. scrobiculata, and $U$. serpens $s$. lat.) were studied.

Phylogenetic analyses provided strong support for the polyphyly of the leaf-stripe smuts within Ustilago. However, the multilocus-based phylogenetic trees support the monophyly of the $U$. striiformis species complex, in contrast to the analysis by Savchenko et al. (2014a), where it was concluded that the $U$. striiformis group was polyphyletic and the segregation of two species was necessary to render it monophyletic. That interpretation was mainly based on a combined LSU-ITS tree of $U$. striiformis species, where $U$. bromina and $U$. nunavutica were located outside the $U$. striiformis s. lat. clade. Because of this conflicting result, the ITS region of the type specimen of $U$. bromina was sequenced (Table 1) and compared with the deposited GenBank sequences of Savchenko et al. (2014a). The type specimen of $U$. bromina on Bromus inermis had an ITS sequence nearly identical (except for a base exchange in a poly $A / T$ region) with the other specimens identified as this species in the current study. It differed in nine bases compared to the three sequences labelled as U. bromina in Savchenko et al. (2014a). It is conceivable that these specimens belong to another undescribed smut species (the three sequences were obtained from material from Israel and USA, while the type collection was from Germany), or the quality of the sequences was not optimal; almost all differences in the sequences from Savchenko et al. (2014a) in comparison to the sequences from this study were located behind a poly $\mathrm{A} / \mathrm{T}$ site, which necessitated re-sequencing for several of the specimens used in this study. Furthermore, misidentification of the host plant seems also possible, as no records were found for the occurrence of Bromus inermis in the floras of Israel (http://flora.org.il/en/plants/) or Palestine (Feinbrun-Dothan 1986).

Ustilago nunavutica was the second species that led Savchenko et al. (2014a) to assume that the U. striformis species complex was polyphyletic. Comparing the ITS and LSU sequences of $U$. nunavutica with sequences from the current study, the LSU sequence used by Savchenko et al. (2014a) showed several SNPs (data not shown), while all other $U$. striiformis samples investigated in this study were identical in the LSU region. In contrast, the ITS sequence of $U$. nunavutica has only few SNPs in comparison to other members of the $U$. striiformis species complex and is identical with $U$. neocopinata. It seems possible that the LSU sequence of $U$. nunavutica either was of bad quality or shows the amplification of a contaminant smut fungus. However, as the genera Puccinellia and Dactylis are not closely related (Schneider et al. 2009) and very high host specificity has been revealed for the closely related species of the $U$. striiformis species complex in this study, it is unlikely that $U$. neocopinata and $U$. nunavutica are conspecific.

In agreement with Stoll et al. (2005) and Spooner \& Legon (2006), we found that $U$. calamagrostidis and $U$. corcontica belonged to the $U$. striiformis species complex. However, further resolution within the $U$. striiformis species complex was only achieved when the protein-coding loci introduced by Kruse et al. (2017b) were employed. The trees revealed a host genus or host species specific occurrence for almost all lineages within the $U$. striiformis species complex, thus they should be treated as distinct species, supported by the observations of Liro (1924). All specimens from a single host species formed a clade according to the host species (or the host genus, in case of Holcus), with the exception of the rather closely related species Lolium perenne and Festuca arundinacea (Malik \& Thomas 1966, Catalán et al. 2004, Hand et al. 2010). As most of these clades received high to 
maximum support, they should be considered to represent distinct species, which can be distinguished based on the host and diagnostic SNPs (Fig. 6). For most of the 14 lineages of the $U$. striiformis species complex validly published names are available, necessitating the description of only two new species in this complex, U. neocopinata on Dactylis glomerata and U. jagei on Agrostis stolonifera s. lat. Vánky (2012) and Savchenko et al. (2014a) mentioned that different species on different hosts within this complex vary remarkably in spore shape, size, and ornamentation. However, morphological variation was observed to be high even within the same host species in the current study and also by Vánky (2012). Thus it is difficult to distinguish these closely related species based on morphology, necessitating the consideration of hosts and SNPs for diagnosis. The host range of at least two species of Ustilago parasitic to Agrostis could not be inferred with certainty, as both ITS and chloroplast loci did not resolve closely related species in the $A$. stolonifera and $A$. gigantea clusters (Amundsen \& Warnke 2012).

While investigating synonymies of the $U$. striiformis species complex, it was found that the name $U$. salweyi is the correct name for the stripe smut on Holcus lanatus. Stevenson (1946) flagged U. salweyi as a "nomen ambiguum", although no action was taken to formally reject the name. Following the ICN (McNeill et al. 2012), the name U. salweyi has priority over Uredo striiformis as it was published two years earlier (Berkeley \& Broome 1850: 463). Although the group generally referred to as the $U$. striiformis-group does not contain a species with that as the correct name, as it is still included as a synonym we feel that it is best to continue to use " $U$. striiformis-group" or "species complex" for these fungi as it is so well established and recalls the symptoms all species of the complex exhibit, although this feature is shared by some leaf-stripe smuts not belonging to this complex.

The species within the $U$. striformis species complex have sometimes been recognised as special forms based on infection trials (Liro 1924, Davis 1930, 1935, Fischer 1940). However, it has been shown for various biotrophic pathogens that the special form concept, in which there is a population continuum with somewhat specialised forms, cannot be upheld (Göker et al. 2004, Lutz et al. 2005, Kemler et al. 2009, Thines et al. 2009, Ploch et al. 2011, Savchenko et al. 2014b, Choi \& Thines 2015).

Similar to the situation in the $U$. striiformis species complex, Ustilago serpens s. lat. on different hosts clustered in phylogenetically distinct subgroups. As the type host for $U$. serpens is Elymus repens, the collections from Bromus inermis warrants recognition as a new species. Ustilago serpens is another example illustrating the narrow host specialization among smut fungi. As for both the coarsely ornamented stripe-smuts ( $U$. serpens clade) and the finely ornamented stripe smuts ( $U$. striiformis clade) only a subset of the known hosts could be included in the current study. It is therefore conceivable that some older names published for specific host-pathogen combinations in these groups warrant recognition and several new species await discovery.

With respect to the global phylogeny of Ustilago it is noteworthy that even based on nine loci the backbone of the phylogenetic tree was only poorly resolved. Conflicting supported topologies were inferred with respect to the phylogenetic position of $U$. maydis in the reconstructions based on three (sister to a clade comprising, among others, the $U$. nuda and the $U$. salweyi clade) and nine loci (sister to a clade comprising the majority of smuts on panicoid grasses).

This highlights the high degree of uncertainty that there still is with respect to the global phylogeny of Ustilago s. lat. (Thines 2016). Considering the diversity of anatomical characteristics and disease syndromes caused, many of which have arisen several times independently (such as the stripe-smut habit; McTaggart et al. 2012a, b, c), any splitting of Ustilago s. lat. into smaller genera as suggested by McTaggart et al. (2012a, 2016) is probably premature and might become obsolete due to the high degree of parallel evolution and associated homoplasy.

\section{ACKNOWLEDGEMENTS}

This study was funded by the LOEWE research funding programme in the framework of the Cluster for Integrative Fungal Research (IPF). We are grateful to the curators of B (Berlin), GLM (Görlitz), $M$ (Munich), and TUB (Tübingen) for allowing the investigation of specimens in their keeping, and David Hawksworth for important information on Ustilago salweyi types in K-M (Kew fungarium) and nomenclatural corrections. We furthermore want to thank the curators of B (Berlin), HBG (Hamburg) and L (Leiden) for searching for the type collection of Ustilago scaura. We thank the private collectors Horst Jage and Volker Kummer for allowing us to include some of their collections in this study. We want to thank Bagdevi Mishra for the opportunity to use TrEase (thines-lab@senckenberg.de/trease) for phylogenetic analysis. Furthermore, we are grateful to Y.-J. Choi for help with the primer design for an internal ITS primer and to Reuel Bennett for proof-reading an initial draft of the manuscript. MT and JK conceived the study; JK made collection trips; FK, HJ, HR, HZ, UR, WD, and VK provided material; JK performed laboratory experiments and microscopy, and also analysed the data; JK and MT interpreted the data and prepared the manuscript with contributions from the other authors.

\section{REFERENCES}

Amundsen K, Warnke S (2012) Agrostis species relationships based on trnL-trnF and atpl-atpH Intergenic Spacer Regions. Hortscience 47: 18-24.

Begerow D, Stoll M, Bauer R (2006) A phylogenetic hypothesis of Ustilaginomycotina based on multiple gene analyses and morphological data. Mycologia 98: 906-916.

Berkeley MJ, Broome CE (1850) Notices of British fungi. Annals and Magazine of Natural History 5: 455-467.

Catalán P, Torrecilla P, Ángel J, Rodríguez L, Olmstead RG (2004) Phylogeny of the festucoid grasses of subtribe Loliinae and allies (Poeae, Pooideae) inferred from ITS and trnL-F sequences. Molecular Phylogenetics and Evolution 31: 517-541.

Choi Y-J, Thines M (2015) Host jumps and radiation, not codivergence drives diversification of obligate pathogens: a case study in downy mildews and Asteraceae. PLOS ONE 10(7): e0133655.

Davis WH (1930) Two physiologic forms of Ustilago striaeformis (Westd.) Niessl. Phytopathology 20: 65-74. 
Davis WH (1935) Summary of investigations with Ustilago striaeformis parasitizing some common grasses. Phytopathology 25: 810-817.

Denchev C, Giraud T, Hood ME (2009) Three new species of anthericolous smut fungi on Caryophyllaceae. Mycologia Balcanica 6: 79-84.

Feinbrun-Dothan N (1986) Flora Palaestina. Vol. 4. Jerusalem: Israel Academy of Sciences and Humanities, Section of Sciences.

Fischer GW (1940) Fundamental studies on the stripe smut of grasses (Ustilago striaeformis) in the Pacific Northwest. Phytopathology 30: 93-118.

Fischer GW (1953) Manual of the North American Smut Fungi. New York: Ronald Press.

Göker M, Riethmüller A, Voglmayr H, Weiss M, Oberwinkler F (2004) Phylogeny of Hyaloperonospora based on nuclear ribosomal internal transcribed spacer sequences. Mycological Progress 3: 83-94.

Guo L (1993) Ustilago deyeuxiae sp. nov. and three smut species new to China. Mycosystema 6: 51-55.

Hand ML, Cogan NOC, Stewart AV, Forster JW (2010) Evolutionary history of tall fescue morphotypes inferred from molecular phylogenetics of the Lolium-Festuca species complex. BMC Evolutionary Biology 10: 303.

Heller A, Thines M (2009) Evidence for the importance of enzymatic digestion of epidermal walls during subepidermal sporulation and pustule opening in white blister rusts (Albuginaceae). Mycological Research 113: 657-667.

Katoh K, Standley DM (2013) MAFFT multiple sequence alignment software version 7: improvements in performance and usability. Molecular Biology and Evolution 30: 772-780.

Kemler M, Lutz M, Göker M, Oberwinkler F, Begerow D (2009) Hidden diversity in the non-caryophyllaceous plant-parasitic members of Microbotryum (Pucciniomycotina: Microbotryales). Systematics and Biodiversity 7: 297-306.

Kruse J, Choi Y-J, Thines M (2017a) New smut-specific primers for the ITS barcoding of Ustilaginomycotina. Mycological Progress 16: 213-221.

Kruse J, Mishra B, Choi Y-J, Sharma R, Thines M (2017b) New smutspecific primers for multilocus genotyping and phylogenetics of Ustilaginaceae. Mycological Progress 16: 1-9.

Liro Jl (1924) Die Ustilagineen Finnlands. Annales Academiae Scientiarum Fennicae, series A 17(1): 1-636.

Lutz M, Göker M, Piątek M, Kemler M, Begerow D, Oberwinkler F (2005) Anther smuts of Caryophyllaceae: molecular characters indicate host-dependent species delimitation. Mycological Progress 4: 225-238.

Malik CP, Thomas PT (1966) Karyotypic studies in some Lolium and Festuca species. Caryologia 19: 167-196.

McNeill J, Barrie FR, Buck WR, Demoulin V, Greuter W, et al. (2012) International Code of Nomenclature for algae, fungi, and plants (Melbourne Code) adopted by the Eighteenth International Botanical Congress Melbourne, Australia, July 2011. [Regnum Vegetabile No. 154.] Königstein: Koeltz Scientific Books.

McTaggart AR, Shivas RG, Geering AD, Callaghan B, Vánky K, Scharaschkin T (2012a) Soral synapomorphies are significant for the systematics of the Ustilago-Sporisorium-Macalpinomyces complex (Ustilaginaceae). Persoonia 29: 63-77.

McTaggart AR, Shivas RG, Geering ADW, Vánky K, Scharaschkin T (2012b) A review of the Ustilago-Sporisorium-Macalpinomyces complex. Persoonia 29: 55-62.
McTaggart AR, Shivas RG, Geering ADW, Vánky K, Scharaschkin T (2012c) Taxonomic revision of Ustilago, Sporisorium and Macalpinomyces. Persoonia 29: 116-132.

McTaggart AR, Shivas RG, Boekhout T, Oberwinkler F, Vánky $\mathrm{K}$, et al. (2016) Mycosarcoma (Ustilaginaceae), a resurrected generic name for corn smut (Ustilago maydis) and its close relatives with hypertrophied, tubular sori. IMA Fungus 7: 309-315.

O'Donnell K (1993) Fusarium and its near relatives. In: The Fungal Holomorph: mitotic, meiotic and pleomorphic speciation in fungal systematics (Reynolds DR, Taylor JW, eds.): 225-233. Wallingford: $C A B$ International.

Piątek M, Lutz M, Chater AO (2013) Cryptic diversity in the Antherospora vaillantii complex on Muscari species. IMA Fungus 4: 5-19.

Ploch S, Telle S, Choi Y-J, Cunnington J, Priest M, et al. (2011) The molecular phylogeny of the white blister rust genus Pustula reveals a case of underestimated biodiversity with several undescribed species on ornamentals and crop plants. Fungal Biology 115: 214-219.

Ridgway KP, Duck JM, Young JPW (2003) Identification of roots from grass swards using PCR-RFLP and FFLP of the plastid trnL (UAA) intron. BMC Ecology 3(8): 1-6.

Ronquist F, Huelsenbeck JP (2003) MRBAYES 3: Bayesian phylogenetic inference under mixed models. Bioinformatics 19: 1572-1574.

Savchenko, KG, Carris LM, Castlebury LA, Heluta VP, Wasser SP, Nevo E (2014a) Stripe smuts of grasses: one lineage or high levels of polyphyly? Persoonia 33: 169-181.

Savchenko KG, Carris LM, Castlebury LA, Heluta VP, Wasser SP, Nevo E (2014b) Revision of Entyloma (Entylomatales, Exobasidiomycetes) on Eryngium. Mycologia 106: 797-810.

Schneider J, Döring E, Hilu KW, Röser M (2009) Phylogenetic structure of the grass subfamily Pooideae based on comparison of plastid matK gene-3'trnK exon and nuclear ITS sequences. Taxon 58: 405-424.

Shenglian L, Wenli C, Phillips SM (2006) 87. Deyeuxia Clarion ex P. Beauvois, Ess. Agrostogr. 43. 1812. In: Flora of China (Zhengyi W, Raven PH, Deyuan H, eds) 22: 348-359. Beijing: Science Press.

Spooner BM, Legon NW (2006) Additions and amendments to the list of British smut fungi. Mycologist 20: 90-96.

Stamatakis A (2014) RAxML version 8: a tool for phylogenetic analysis and post-analysis of large phylogenies. Bioinformatics 30: 1312-1313.

Stevenson JA (1946) A nomenclatorial discussion of Ustilago striiformis. Plant Disease Reporter 30: 53-59.

Stoll M, Begerow D, Oberwinkler F (2005) Molecular phylogeny of Ustilago, Sporisorium, and related taxa based on combined analyses of rDNA sequences. Mycological Research 109: 342356.

Stoll M, Piepenbring M, Begerow D, Oberwinkler F (2003) Molecular phylogeny of Ustilago and Sporisorium species (Basidiomycota, Ustilaginales) based on internal transcribed spacer (ITS) sequences. Canadian Journal of Botany 81: 976-984.

Tamura K, Stecher G, Peterson D, Filipski A, Kumar S (2013) MEGA6: Molecular Evolutionary Genetics Analysis Version 6.0. Molecular Biology and Evolution 30: 2725-2729.

Thines M (2016) Proposal to conserve the name Ustilago (Basidiomycota) with a conserved type. Taxon 65: 1170-1171.

Thines M, Choi Y-J, Kemen E, Ploch S, Holub EB, Shin H-D, Jones JDG (2009) A new species of Albugo parasitic to Arabidopsis 
thaliana reveals new evolutionary patterns in white blister rusts (Albuginaceae). Persoonia 22: 123-128.

Vánky K (2012) Smut Fungi of the World. St Paul, MN: American Phytopathological Society Press.

Vánky K (2007) Taxonomic studies on Ustilaginomycetes - 27. Mycotaxon 99: 1-70.
White TJ, Bruns T, Lee S, Taylor J (1990) Amplification and direct sequencing of fungal ribosomal RNA sequences for phylogenetics. In: PCR Protocols: a guide to methods and applications (Innis N, Gelfand D, Sninsky J, White T, eds): 315322. San Diego: Academic Press. 